\title{
Transverse oscillation of particles in the vicinity of resonances for a cyclotron
}

\author{
Kai Zhou $\odot,{ }^{1,2}$ Yuntao Song, ${ }^{1,2}$ Gen Chen, ${ }^{1, *}$ Kaizhong Ding, ${ }^{1, \dagger}$ and Galina Karamysheva ${ }^{3}$ \\ ${ }^{1}$ Institute of Plasma Physics, Chinese Academy of Sciences, Hefei 230000, China \\ ${ }^{2}$ University of Science and Technology of China, Hefei 230000, China \\ ${ }^{3}$ Joint Institute for Nuclear Research, Dubna 41980, Russia
}

(Received 24 April 2019; published 17 October 2019)

\begin{abstract}
Transverse oscillation is an important issue in beam dynamics of cyclotrons and can be described by the Mathieu equation. We review the standard form of the Mathieu equation, $\frac{d^{2} u}{d \theta^{2}}+(\delta+\varepsilon \cdot \cos 2 \theta) u=0$, and propose a modification of the method of multiple scales (i.e., a perturbation method) so that the asymptotic analytical solutions of the Mathieu equation can be computed in the stable and unstable regions for both $\delta \geq 0$ and $\delta<0$. This method was applied to the nonlinear transverse oscillation equations for a cyclotron. Analytical solutions for transverse oscillation in the stable and unstable regions (i.e., vicinity of the resonances) were obtained, and the accuracy of these analytical solutions was confirmed by their close agreement with the direct numerical integration. Useful results such as the analytical solution of the transverse oscillation frequency, increasing rate of the amplitude in unstable regions, and the resonance width were also derived; the stable condition and driving terms of the resonances can be obtained from the analytical solutions.
\end{abstract}

DOI: 10.1103/PhysRevAccelBeams.22.104001

\section{INTRODUCTION}

The well-known Mathieu equation has the form of $\frac{d^{2} u}{d \theta^{2}}+(\delta+\varepsilon \cdot \cos 2 \theta) u=0$; it occurs in a wide variety of physical problems and has been widely investigated since its discovery. The solution to the Mathieu equation is determined by its coefficients. The $\delta-\varepsilon$ plane is separated into stable and unstable regions by transition curves. The parameter pairs $(\delta, \varepsilon)$ that belong to the stable regions correspond to bounded solutions; parameter pairs $(\delta, \varepsilon)$ that belong to the unstable regions correspond to unbounded solutions [1-5].

Perturbation methods such as the method of multiple scales (MMS) and Lindstedt-Poincaré (LP) method are effective at solving the transition curves and deriving analytical solutions for the Mathieu equation but have some limitations. The LP method can only obtain bounded asymptotic solutions for stable regions, not unbounded solutions for unstable regions [6-10]. The MMS can obtain asymptotic solutions for both stable and

\footnotetext{
*chengen@ipp.ac.cn

'kzding@ipp.ac.cn
}

Published by the American Physical Society under the terms of the Creative Commons Attribution 4.0 International license. Further distribution of this work must maintain attribution to the author(s) and the published article's title, journal citation, and DOI. unstable regions in the case of $\delta \geq 0$ but is invalid when $\delta<0$ [7-11]. In this paper, we propose a modified MMS that can obtain the bounded and unbounded solutions for not only $\delta \geq 0$ but also $\delta<0$.

To date, numerical simulations [12-18] are widely used to study transverse oscillations in cyclotrons. Although they can accurately describe the particle motion, they cannot intuitively show the relationship between the parameters of a magnetic field and particle motion behavior in the vicinity of resonance. There are also some analytical formulas describing transverse oscillation as well as resonances for cyclotrons in earlier years in Refs. [19-23]. In this paper, we applied the modified MMS to two-dimensional (2D) nonlinear Mathieu-type equations that arise in the study of the transverse oscillations for the SC200 superconducting cyclotron. Analytical solutions for the transverse oscillation in the stable and unstable regions were obtained. For a systematic study, the stable conditions, the driving terms and the amplitude growth in the vicinity of $2 Q_{r}=2, Q_{z}=\frac{1}{2}, Q_{r}-2 Q_{z}=1$ were discussed using the analytical solutions. These analytical results were confirmed by a comparison with the direct numerical integration. As an analytical study, the contribution of this work is a quantitative and accurate analytical interpretation of the transverse oscillation for the cyclotron, and a comprehensive discussion on the resonances, which can help us have a better understanding of the dynamics. 


\section{MODIFIED METHOD OF MULTIPLE SCALES FOR SOLVING THE MATHIEU EQUATION}

\section{A. Standard form of the method of multiple scales}

The standard form of the Mathieu equation is written as

$$
\frac{d^{2} u}{d \theta^{2}}+(\delta+\varepsilon \cdot \cos 2 \theta) u=0,
$$

where $\delta$ and $\varepsilon$ are real numbers. The first step of the MMS is to introduce several scales $\theta_{n}=\varepsilon^{n} \cdot \theta$, where $n=0,1,2, \ldots$. In other words,

$$
\theta_{0}=\theta, \quad \theta_{1}=\varepsilon \cdot \theta, \quad \theta_{2}=\varepsilon^{2} \cdot \theta .
$$

These scales are treated as independent. One consequence is that the derivative with respect to $\theta$ is now transformed into

$$
\begin{gathered}
\frac{d}{d \theta}=D_{0}+\varepsilon \cdot D_{1}+\varepsilon^{2} \cdot D_{2}+\cdots, \\
\frac{d^{2}}{d \theta^{2}}=D_{0}^{2}+\varepsilon \cdot 2 D_{0} D_{1}+\varepsilon^{2} \cdot\left(2 D_{0} D_{2}+D_{1}^{2}\right)+\cdots,
\end{gathered}
$$

where $D_{0}, D_{1}$, and $D_{2}$ are partial differential operators defined as

$$
D_{0}=\frac{\partial}{\partial \theta_{0}}, \quad D_{1}=\frac{\partial}{\partial \theta_{1}}, \quad D_{2}=\frac{\partial}{\partial \theta_{2}} .
$$

According to the MMS, the solution of Eq. (1) has a power series expansion:

$$
\begin{aligned}
u\left(\theta_{0}, \theta_{1}, \theta_{2}\right)= & u_{0}\left(\theta_{0}, \theta_{1}, \theta_{2}\right)+\varepsilon \cdot u_{1}\left(\theta_{0}, \theta_{1}, \theta_{2}\right) \\
& +\varepsilon^{2} \cdot u_{2}\left(\theta_{0}, \theta_{1}, \theta_{2}\right)+\cdots .
\end{aligned}
$$

Substituting Eqs. (3), (4), and (6) into Eq. (1), and letting the coefficients of each power of $\varepsilon$ to be zero, we arrive at the following approximate equations:

$$
\begin{gathered}
D_{0}^{2} u_{0}+\delta \cdot u_{0}=0, \\
D_{0}^{2} u_{1}+2 D_{0} D_{1} \cdot u_{0}+\delta \cdot u_{1}=-\cos 2 \theta \cdot u_{0}, \\
D_{0}^{2} u_{2}+2 D_{0} D_{1} \cdot u_{1}+\left(2 D_{0} D_{2}+D_{1}^{2}\right) \cdot u_{0}+\delta \cdot u_{2} \\
=-\cos 2 \theta \cdot u_{1} .
\end{gathered}
$$

In the above procedure, a differential equation with variable coefficients [Eq. (1)] is transformed into several approximate equations with constant coefficients [Eqs. (7)-(9)]. Each linear partial differential equation in Eqs. (7)-(9) can be solved in sequence. The solution to Eq. (7) is easily found:

$$
u_{0}=E\left(\theta_{1}, \theta_{2}\right) \cdot e^{i \sqrt{\delta} \theta_{0}}+E^{*}\left(\theta_{1}, \theta_{2}\right) \cdot e^{-i \sqrt{\delta} \theta_{0}} .
$$

This is the zeroth-order solution, where the coefficients $E\left(\theta_{1}, \theta_{2}\right)$ and $E^{*}\left(\theta_{1}, \theta_{2}\right)$ are undetermined conjugated functions. Substituting Eq. (10) into Eq. (8) results in

$$
\begin{aligned}
D_{0}^{2} u_{1}+\delta \cdot u_{1}= & -2\left[i \sqrt{\delta} \cdot D_{1} E\left(\theta_{1}, \theta_{2}\right) \cdot e^{i \sqrt{\delta} \theta_{0}}\right. \\
& \left.-i \sqrt{\delta} \cdot D_{1} E^{*}\left(\theta_{1}, \theta_{2}\right) \cdot e^{-i \sqrt{\delta} \theta_{0}}\right] \\
& -\frac{1}{2}\left(e^{2 i \theta_{0}}+e^{-2 i \theta_{0}}\right) \\
& \cdot\left[E\left(\theta_{1}, \theta_{2}\right) \cdot e^{i \sqrt{\delta} \theta_{0}}+E^{*}\left(\theta_{1}, \theta_{2}\right) \cdot e^{-i \sqrt{\delta} \theta_{0}}\right] .
\end{aligned}
$$

The inhomogeneous terms [i.e., right side of Eq. (11)] contain

$$
\begin{aligned}
-2 & \left\lfloor i \sqrt{\delta} \cdot D_{1} E\left(\theta_{1}, \theta_{2}\right) \cdot e^{i \sqrt{\delta} \theta_{0}}\right. \\
& \left.-i \sqrt{\delta} \cdot D_{1} E^{*}\left(\theta_{1}, \theta_{2}\right) \cdot e^{-i \sqrt{\delta} \theta_{0}}\right\rfloor,
\end{aligned}
$$

which has the same frequency as the associated homogeneous equation. This causes secular terms, which must be avoided. Therefore, the coefficients of $e^{i \sqrt{\delta} \theta_{0}}$ and $e^{-i \sqrt{\delta} \theta_{0}}$ in Eq. (12) need to be zero. Eliminating these coefficients yields the condition for determining the undetermined function $E\left(\theta_{1}, \theta_{2}\right)$ :

$$
\begin{gathered}
D_{1} E\left(\theta_{1}, \theta_{2}\right)=\frac{\partial E\left(\theta_{1}, \theta_{2}\right)}{\partial \theta_{1}}=0, \\
D_{1} E^{*}\left(\theta_{1}, \theta_{2}\right)=\frac{\partial E^{*}\left(\theta_{1}, \theta_{2}\right)}{\partial \theta_{1}}=0 .
\end{gathered}
$$

In other words, $E\left(\theta_{1}, \theta_{2}\right)$ and $E^{*}\left(\theta_{1}, \theta_{2}\right)$ are independent of $\theta_{1}$ and are functions of $\theta_{2}$ :

$$
E\left(\theta_{1}, \theta_{2}\right)=E\left(\theta_{2}\right), \quad E^{*}\left(\theta_{1}, \theta_{2}\right)=E^{*}\left(\theta_{2}\right) .
$$

Equation (13) represents the condition for avoiding the secular terms in $u_{1}$. After the secular terms are removed, the solution of Eq. (11) can be written as

$$
\begin{aligned}
u_{1}= & -\frac{1}{2}\left[\frac{E\left(\theta_{2}\right)}{\delta-(\sqrt{\delta}+2)^{2}} \cdot e^{i(\sqrt{\delta}+2) \theta_{0}}\right. \\
& +\frac{E\left(\theta_{2}\right)}{\delta-(\sqrt{\delta}-2)^{2}} \cdot e^{i(\sqrt{\delta}-2) \theta_{0}} \\
& +\frac{E^{*}\left(\theta_{2}\right)}{\delta-(\sqrt{\delta}+2)^{2}} \cdot e^{-i(\sqrt{\delta}+2) \theta_{0}} \\
& \left.+\frac{E^{*}\left(\theta_{2}\right)}{\delta-(\sqrt{\delta}-2)^{2}} \cdot e^{-i(\sqrt{\delta}-2) \theta_{0}}\right] .
\end{aligned}
$$


This is the first-order solution. By substituting Eq. (15) into Eq. (9) and letting the secular terms be zero, we obtain the condition for avoiding secular terms in $u_{2}$ :

$\frac{\partial E\left(\theta_{2}\right)}{\partial \theta_{2}}=-i \Lambda_{1} \cdot E\left(\theta_{2}\right), \quad \frac{\partial E^{*}\left(\theta_{2}\right)}{\partial \theta_{2}}=i \Lambda_{1} \cdot E^{*}\left(\theta_{2}\right)$,

where

$$
\Lambda_{1}=\frac{\left[\frac{1}{\delta-(2+\sqrt{\delta})^{2}}+\frac{1}{\delta-(2-\sqrt{\delta})^{2}}\right]}{8 \sqrt{\delta}} .
$$

Equation (16) represents the condition to determine $E\left(\theta_{2}\right)$. Then, $E\left(\theta_{2}\right)$ and $E^{*}\left(\theta_{2}\right)$ can be expressed as

$$
E\left(\theta_{2}\right)=C_{1} \cdot e^{-i \Lambda_{1} \theta_{2}}, \quad E^{*}\left(\theta_{2}\right)=C_{1}^{*} \cdot e^{i \Lambda_{1} \theta_{2}},
$$

where $C_{1}$ and $C_{1}^{*}$ are conjugated complex numbers that are determined by the initial condition. With the above procedure, the undetermined conjugated complex functions $E\left(\theta_{1}, \theta_{2}\right)$ and $E^{*}\left(\theta_{1}, \theta_{2}\right)$ are completely determined, and $u_{0}\left(\theta_{0}, \theta_{1}, \theta_{2}\right)$ and $u_{1}\left(\theta_{0}, \theta_{1}, \theta_{2}\right)$ are determined next. Finally, by substituting $u_{0}$ and $u_{1}$ into Eq. (6), the solution to the original Eq. (1) is given by

$$
\begin{aligned}
u= & u_{0}+\varepsilon \cdot u_{1}+\cdots \\
= & C_{1} \cdot e^{i\left(-\varepsilon^{2} \cdot \Lambda_{1}+\sqrt{\delta}\right) \theta}+C_{1}^{*} \cdot e^{-i\left(-\varepsilon^{2} \cdot \Lambda_{1}+\sqrt{\delta}\right) \theta} \\
& -\frac{\varepsilon}{2}\left[\frac{E}{\delta-(\sqrt{\delta}+2)^{2}} \cdot e^{i(\sqrt{\delta}+2) \theta}+\frac{E}{\delta-(\sqrt{\delta}-2)^{2}} \cdot e^{i(\sqrt{\delta}-2) \theta}+\frac{E^{*}}{\delta-(\sqrt{\delta}+2)^{2}} \cdot e^{-i(\sqrt{\delta}+2) \theta}+\frac{E^{*}}{\delta-(\sqrt{\delta}-2)^{2}} \cdot e^{-i(\sqrt{\delta}-2) \theta}\right] .
\end{aligned}
$$

The perturbed frequency of the system can be written as

$$
Q_{r}=-\varepsilon^{2} \cdot \Lambda_{1}+\sqrt{\delta} .
$$

The numerical solution of Eq. (1) for $\delta \geq 0$ is obtained with the fourth-order Runge-Kutta method and can be used to validate the accuracy of the analytical solutions. Figure 1 compares the results of the above analytical formulas and numerical integration. The differences in the trajectories are almost indistinguishable, and the difference in the phase motion is very small. This proves that the above analytical formulas are accurate.

\section{B. Modification of the method of multiple scales}

As shown in Eq. (7), the zeroth-order approximation equation has no periodic solutions when $\delta<0$; that is, the MMS is invalid for this case. In fact, much literature on nonlinear dynamics has applied the MMS to the transition curves and asymptotic solutions of the Mathieu equation with $\delta \geq 0$, but applying the MMS in the case of $\delta<0$ has not been commonly discussed. We present a simple but effective modification of the MMS to obtain both the bounded and unbounded solutions for the Mathieu equation with $\delta<0$.
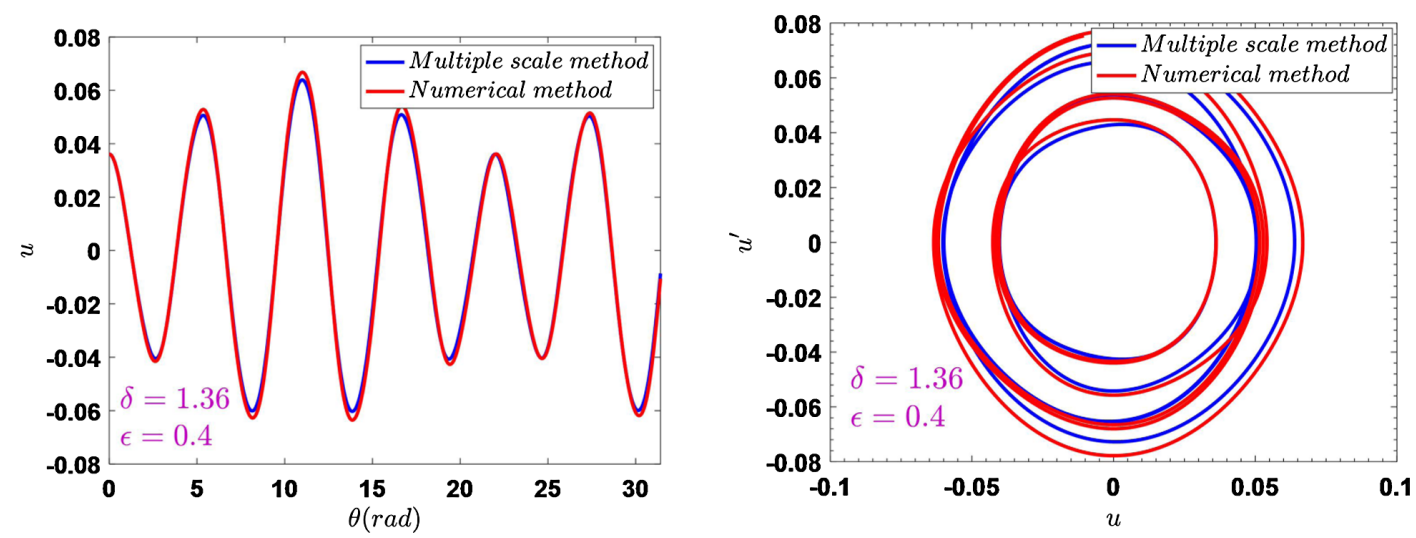

FIG. 1. Trajectories (left) and phase motions (right) for parameter pairs $(\delta, \varepsilon)=(1.36,0.4)$ in the stable region. The MMS is in blue, and the direct numerical integration is in red. 
Equation (1) contains the linear term $\delta \cdot u$ and the flutter term $\varepsilon \cdot \cos 2 \theta \cdot u$. For $\delta \geq 0$, the flutter term is a higher order small quantity than the linear term when $\varepsilon$ is a small parameter. We can obtain the corresponding generating system $\frac{d^{2} u}{d \theta^{2}}+\delta \cdot u=0$ by setting $\varepsilon=0$; this is a stable simple harmonic system, the flutter term could be treated as a perturbation to this system. However, for the case of $\delta<0$, the problem has changed in nature. The previous generating system becomes unstable due to the linear term, and the stability of the original system is mainly dependent on the flutter term. At this point, we take the linear term as a higher order smaller quantity than the flutter term; then, the new generating system becomes $\frac{d^{2} u}{d \theta^{2}}=0$. To this end, we introduce a new small parameter that satisfies $0 \leq \xi \leq 1$ to distinguish the perturbations of different orders, and a higher order small parameter is introduced to a smaller perturbation term:

$$
\frac{d^{2} u}{d \theta^{2}}+\xi^{3} \cdot \delta \cdot u=-\xi \cdot \varepsilon \cdot \cos 2 \theta \cdot u .
$$

The original small parameter $\varepsilon$ is now treated as a constant. Equation (21) is equivalent to Eq. (1) when $\xi=1$. After Eq. (21) is solved, the solution of Eq. (1) can be obtained simply by letting $\xi=1$. The details for solving Eq. (21) are presented below.

By expanding the variable $u$ and derivative operator $\frac{d^{2}}{d \theta^{2}}$ with respect to the new small parameter $\xi$ and letting the coefficients of each power of $\xi$ be zero, we obtain the following approximate equations:

$$
\begin{gathered}
D_{0}^{2} u_{0}=0 \\
D_{0}^{2} u_{1}+2 D_{0} D_{1} u_{0}=-\varepsilon \cdot \cos 2 \theta \cdot u_{0}, \\
D_{0}^{2} u_{2}+2 D_{0} D_{1} u_{1}+\left(2 D_{0} D_{2}+D_{1}^{2}\right) u_{0}=-\varepsilon \cdot \cos 2 \theta \cdot u_{1}, \\
D_{0}^{3} u_{3}+2 D_{0} D_{1} u_{2}+\left(2 D_{0} D_{2}+D_{1}^{2}\right) u_{1} \\
+\left(2 D_{0} D_{3}+2 D_{1} D_{2}\right) u_{0}+\delta \cdot u_{0}=-\varepsilon \cdot \cos 2 \theta \cdot u_{2} .
\end{gathered}
$$

Here, the zeroth-order and first-order approximate equations do not contain secular terms; the zeroth-order and first-order solutions can be solved from Eqs. (22) and (23) directly:

$$
\begin{gathered}
u_{0}=F\left(\theta_{1}, \theta_{2}\right), \\
u_{1}=\frac{\varepsilon}{8} \cdot\left(e^{2 i \theta_{0}}+e^{-2 i \theta_{0}}\right) \cdot F\left(\theta_{1}, \theta_{2}\right),
\end{gathered}
$$

where $F\left(\theta_{1}, \theta_{2}\right)$ is an undetermined function. Then the second- and third-order solutions can be solved from
Eqs. (24) and (25), but higher-order solutions are usually very small and thus can be neglected. However, we cannot determine the solution completely with only the first two order equations because the zeroth- and first-order solutions contain the undetermined coefficients $F\left(\theta_{1}, \theta_{2}\right)$, which have to be determined by eliminating the secular terms in Eqs. (24) and (25).

Substituting the first-order solution [Eq. (27)] into the second-order approximate equation [Eq. (24)] and eliminating the secular terms yields the condition for determining $F\left(\theta_{1}, \theta_{2}\right)$ :

$$
D_{1}^{2} F\left(\theta_{1}, \theta_{2}\right)=-\frac{\varepsilon^{2}}{8} \cdot F\left(\theta_{1}, \theta_{2}\right) .
$$

For simplification, we let

$$
\Lambda_{2}^{2}=\frac{\varepsilon^{2}}{8} .
$$

Then, $F\left(\theta_{1}, \theta_{2}\right)$ can be expressed as

$$
F\left(\theta_{1}, \theta_{2}\right)=\psi\left(\theta_{2}\right) \cdot e^{i \Lambda_{2} \theta_{1}}+\psi^{*}\left(\theta_{2}\right) \cdot e^{-i \Lambda_{2} \theta_{1}},
$$

where $\psi\left(\theta_{2}\right)$ is an undetermined function. After the secular terms in Eq. (25) are eliminated, the second-order solution $u_{2}$ follows. In general, however, the form of the second- or higher-order approximation is very complicated, and the corrections they provide are negligible. Thus, these highorder approximate equations are not considered in further derivations. That is,

$$
u_{2}=0, \quad u_{3}=0 .
$$

By substituting $u_{2}=0$ into Eq. (25) and eliminating the secular term, we obtain the condition for determining $\psi\left(\theta_{2}\right)$ :

$$
\begin{gathered}
2\left\{\left(i \Lambda_{2}\right) \frac{\partial \psi\left(\theta_{2}\right)}{\partial \theta_{2}} e^{i \Lambda_{2} \theta_{1}}+\left(-i \Lambda_{2}\right) \frac{\partial \psi^{*}\left(\theta_{2}\right)}{\partial \theta_{2}} e^{-i \Lambda_{2} \theta_{1}}\right\} \\
+\delta \cdot\left[\psi\left(\theta_{2}\right) \cdot e^{i \Lambda_{2} \theta_{1}}+\psi^{*}\left(\theta_{2}\right) \cdot e^{-i \Lambda_{2} \theta_{1}}\right]=0 .
\end{gathered}
$$

The solution of Eq. (32) is

$$
\begin{aligned}
\psi\left(\theta_{2}\right) & =C_{2} \cdot \exp \left(i \frac{\delta}{2 \Lambda_{2}} \theta_{2}\right), \\
\psi^{*}\left(\theta_{2}\right) & =C_{2}^{*} \cdot \exp \left(-i \frac{\delta}{2 \Lambda_{2}} \theta_{2}\right),
\end{aligned}
$$

where $C_{2}$ and $C_{2}^{*}$ are complex conjugated numbers determined by the initial condition. If Eq. (33) is substituted into Eq. (30), $F\left(\theta_{1}, \theta_{2}\right)$ can be expressed as

$$
F\left(\theta_{1}, \theta_{2}\right)=C_{2} \cdot e^{i\left(\Lambda_{2} \theta_{1}+\frac{\delta}{2 \Lambda_{2}} \theta_{2}\right)}+C_{2}^{*} \cdot e^{-i\left(\Lambda_{2} \theta_{1}+\frac{\delta}{2 \Lambda_{2}} \theta_{2}\right)} .
$$


With the above procedure, the undetermined function $F\left(\theta_{1}, \theta_{2}\right)$ is completely determined, following which $u_{0}$ and $u_{1}$ are determined. Finally, the solution of Eq. (1) can be obtained by letting $\xi=1$ :

$$
\begin{aligned}
u= & u_{0}+\xi \cdot u_{1}+\cdots=u_{0}+u_{1}+\cdots \\
= & \left\{C_{2} \cdot e^{i\left(\Lambda_{2}+\frac{\delta}{2 \Lambda_{2}}\right) \theta}+C_{2}^{*} \cdot e^{-i\left(\Lambda_{2}+\frac{\delta}{2 \Lambda_{2}}\right) \theta}\right\} \\
& \cdot\left\{1+\frac{\varepsilon}{8} \cdot\left(e^{2 i \theta}+e^{-2 i \theta}\right)\right\} .
\end{aligned}
$$

The perturbed frequency of the system can be written as

$$
Q_{z}=\Lambda_{2}+\frac{\delta}{2 \Lambda_{2}}
$$

Based on Eq. (29), $\Lambda_{2}$ can be expressed as $\Lambda_{2}=\sqrt{\frac{\varepsilon^{2}}{8}}$; here, $\varepsilon$ is the amplitude of the flutter term. It is noted that Eqs. (35) and (36) are valid for $\Lambda_{2} \neq 0$ because the derivation is based on the assumption that the flutter term is the dominant term and the linear term is a perturbation. Thus, $\Lambda_{2}$ should not be zero; otherwise, the flutter term will vanish, and the assumption will be invalid. Letting $Q_{z}>0$, we obtain $\Lambda_{2}>\sqrt{\frac{-\delta}{2}}$. If $0<\Lambda_{2}<\sqrt{\frac{-\delta}{2}}$, then the flutter term is too small to overcome the instability caused by the linear component; thus, the system is nonoscillating and the frequency does not exist. Only when $\Lambda_{2}>\sqrt{\frac{-\delta}{2}}$, namely the flutter term is sufficiently large, the system can be stable and the corresponding frequency can be described by Eq. (36).

The numerical solution of Eq. (1) for $\delta<0$ obtained with the direct numerical integration (the fourth-order Runge-Kutta method) can be used to validate the accuracy of the analytical solutions. Figure 2 compares the results of the above analytical formulas and those from direct numerical integration. The errors between these two methods were very small, which verifies the accuracy of the above analytical formulas.

In this section, we consider the asymptotic solution of the Mathieu equation for $\delta \geq 0$ and $\delta<0$ in the stable regions. However, obtaining a uniform general solution for the Mathieu equation in the unstable regions is difficult because the forms of the unbounded solutions in the unstable regions strongly depend on the parameter pairs $(\delta, \varepsilon)$. In order to study the solutions in unstable regions, we give several specific examples that come from the beam dynamics of an isochronous cyclotron in the next section.

\section{APPLICATION TO THE BEAM DYNAMICS OF A CYCLOTRON}

The transverse oscillation is also known as the betatron oscillation and is one of the most important issues in beam dynamics [24,25]. It is well known that the transverse oscillation can be unstable when its frequency approaches the resonance condition $n \cdot Q_{r}+m \cdot Q_{z}=l$. In this section, we take the SC200 cyclotron as an example and solve the transverse oscillation equations in its stable and unstable regions (i.e., in the vicinity of resonances) with the MMS. We discuss the resonances in the vicinity of $2 Q_{r}=2, Q_{z}=\frac{1}{2}$, and $Q_{r}-2 Q_{z}=1$, and useful results such as the resonance width, the driving terms, the stable conditions, and increasing rate of amplitude in the unstable regions are derived.

SC200 is a compact superconducting proton cyclotron used for proton therapy. It can accelerate protons to $200 \mathrm{MeV}$ and was designed in collaboration by ASIPP (China) and JINR (Russia) [26]. SC200 has four spiral sectors. The mean magnetic field is about $2.95 \mathrm{~T}$ in the central region and $3.6 \mathrm{~T}$ in the extraction region.
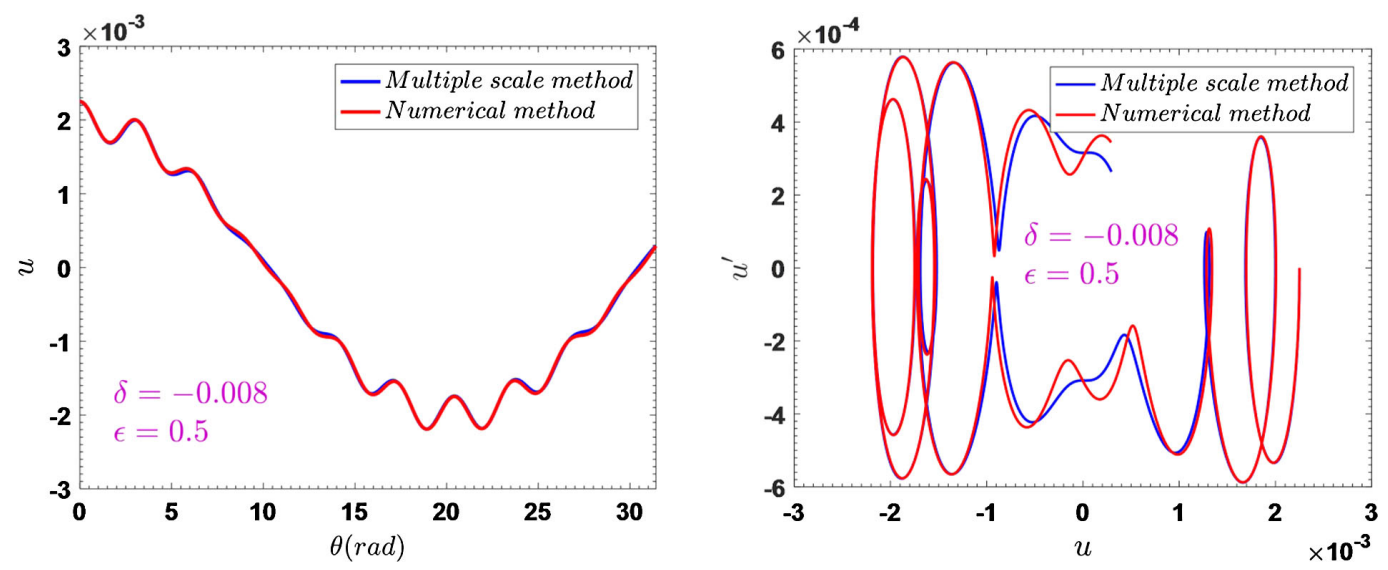

FIG. 2. Trajectories (left) and phase motions (right) for parameter pairs $(\delta, \varepsilon)=(-0.008,0.5)$ in the stable region. The MMS is in blue, and the direct numerical integration is in red. 


\section{A. Transverse oscillation in stable regions}

The small amplitude transverse oscillation in a cyclotron can be described by the following 2D Mathieu-type equations (see the Appendix):

$$
\begin{gathered}
u^{\prime \prime}+Q_{u}^{2} \cdot u=-g(\theta) \cdot u, \\
v^{\prime \prime}+Q_{v}^{2} \cdot v=-h(\theta) \cdot v+J(\theta) \cdot u v,
\end{gathered}
$$

where $u$ corresponds to the radial oscillation and $v$ corresponds to the vertical oscillation. $Q_{u}$ and $Q_{v}$ are unperturbed frequencies that satisfy $Q_{u}^{2}>0$ and $Q_{v}^{2}<0$. $g(\theta), h(\theta)$, and $J(\theta)$ are periodic functions with a period of $T=2 \pi$. The properties of these coefficients are shown in Figs. 10 and 11.

The physical meanings of $Q_{u}$ and $Q_{v}$ are the unperturbed tunes for zero flutter. They are related to the field index of the mean magnetic field component. In an isochronous cyclotron, the mean magnetic field component $B_{0}(r)$ increases with the radius to satisfy the requirement of isochronism. The field index $n=-\frac{r}{B_{0}(r)} \frac{d B_{0}(r)}{d r}$ is negative. In this case, the vertical focusing is provided by the flutter field component, and the mean field component provides vertical defocusing; so, we have $Q_{v}^{2}<0$ in the vertical plane, which corresponds to $\delta<0$ in the Mathieu equation. Thus, the modified MMS is applied to the vertical plane.

Similar to the standard form of the Mathieu equation, the stability of Eqs. (37) and (38) is related to their coefficients [e.g., $Q_{u}^{2}, Q_{v}^{2}, g(\theta)$ ]. However, the relationship is more complex, unlike in the standard form where it is easily distinguished by the transition curves. However, when $Q_{u}^{2}$, $Q_{v}^{2}$, and $g(\theta)$ satisfy a certain relationship and make the actual tunes approach the resonance conditions, the system can be unstable.

$g(\theta)$ and $h(\theta)$ are functions of the equilibrium orbit parameters and can be expanded into Fourier series:

$$
\begin{aligned}
& g(\theta)=\sum_{n} P_{n} e^{i n \theta}+P_{n}^{*} e^{-i n \theta}, \\
& h(\theta)=\sum_{n} M_{n} e^{i n \theta}+M_{n}^{*} e^{-i n \theta} .
\end{aligned}
$$

The values of the Fourier coefficients are proportional to the magnetic field harmonics. First, the small parameter $\varepsilon$ that satisfies $0 \leq \varepsilon \leq 1$ is introduced; then, the transverse oscillation equations, Eqs. (37) and (38), are transformed into the following perturbation equations:

$$
\begin{gathered}
u^{\prime \prime}+Q_{u}^{2} \cdot u=-\varepsilon \cdot g(\theta) \cdot u, \\
v^{\prime \prime}+\varepsilon^{3} \cdot Q_{v}^{2} \cdot v=-\varepsilon \cdot h(\theta) \cdot v+\varepsilon^{2} \cdot J(\theta) \cdot u v .
\end{gathered}
$$

As described in Eq. (21), the principle of introducing small parameters is based on the magnitude of the perturbation; a higher-order small parameter is introduced for a smaller perturbation term. Here, we introduced the small parameters in Eqs. (37) and (38) with reference to Eq. (21). It is noted that Eq. (38) differs from Eq. (21) in that it contains a coupling term $J(\theta) \cdot u v$, which is a second-order small quantity, whereas the flutter term $h(\theta)$. $v$ is a first order small quantity. Thus, $\varepsilon^{2}$ is introduced into the coupling term, and $\varepsilon$ is introduced into the flutter term to distinguish the orders of small quantity.

The key point of the MMS is introducing scaled time coordinates and observing the motion at different timescales. In Eq. (42), the vertical motion is observed at a different timescale, because the radial focusing is much stronger than the vertical focusing owing to the negative field index. Consequently, the vertical motion is much slower and should be observed at a slower timescale. Introducing the cubic power $\varepsilon^{3}$ to the vertical motion corresponds to a much slower timescale.

First, we introduce three scales $\theta_{0}=\theta, \theta_{1}=\varepsilon \cdot \theta$, and $\theta_{2}=\varepsilon^{2} \cdot \theta$. Then, we expand $u$ and $v$ in power series in $\varepsilon$ as follows:

$$
\begin{gathered}
u=u_{0}+\varepsilon \cdot u_{1}+\varepsilon^{2} \cdot u_{2}+\cdots, \\
v=v_{0}+\varepsilon \cdot v_{1}+\varepsilon^{2} \cdot v_{2}+\varepsilon^{3} \cdot v_{3} \cdots .
\end{gathered}
$$

Then, the radial oscillation equation is approximated as follows:

$$
\begin{gathered}
D_{0}^{2} u_{0}+Q_{u}^{2} \cdot u_{0}=0, \\
D_{0}^{2} u_{1}+2 D_{0} D_{1} \cdot u_{0}+Q_{u}^{2} \cdot u_{1}=-g(\theta) \cdot u_{0}, \\
D_{0}^{2} u_{2}+2 D_{0} D_{1} \cdot u_{1}+\left(2 D_{0} D_{2}+D_{1}^{2}\right) \cdot u_{0}+Q_{u}^{2} \cdot u_{2} \\
=-g(\theta) \cdot u_{1} .
\end{gathered}
$$

The vertical oscillation equation is approximated as follows:

$$
\begin{gathered}
D_{0}^{2} v_{0}=0, \\
D_{0}^{2} v_{1}+2 D_{0} D_{1} v_{0}=-h(\theta) \cdot v_{0}, \\
D_{0}^{2} v_{2}+2 D_{0} D_{1} v_{1}+2\left(D_{0} D_{2}+D_{1}^{2}\right) v_{0} \\
=-h(\theta) \cdot v_{1}+J(\theta) \cdot u_{0} v_{0}, \\
D_{0}^{3} v_{3}+2 D_{0} D_{1} v_{2}+\left(2 D_{0} D_{2}+D_{1}^{2}\right) v_{1} \\
+\left(2 D_{0} D_{3}+2 D_{1} D_{2}\right) v_{0}+Q_{v}^{2} v_{0} \\
=-h(\theta) \cdot v_{2}+J(\theta) \cdot\left[u_{0} v_{1}+u_{1} v_{0}\right] .
\end{gathered}
$$

For the radial oscillation, the zeroth-order solution [i.e., solution of Eq. (45)] is easily found: 


$$
u_{0}=E\left(\theta_{1}, \theta_{2}\right) \cdot e^{i Q_{u} \theta_{0}}+E^{*}\left(\theta_{1}, \theta_{2}\right) \cdot e^{-i Q_{u} \theta_{0}} .
$$

The first-order solution [i.e., solution of Eq. (46)] is

$$
\begin{aligned}
u_{1}= & -\sum_{n} \frac{P_{n} E\left(\theta_{1}, \theta_{2}\right)}{Q_{u}^{2}-\left(n+Q_{u}\right)^{2}} \cdot e^{i\left(n+Q_{u}\right) \theta_{0}}+\frac{P_{n} E^{*}\left(\theta_{1}, \theta_{2}\right)}{Q_{u}^{2}-\left(n-Q_{u}\right)^{2}} \\
& \cdot e^{i\left(n-Q_{u}\right) \theta_{0}}+\frac{P_{n}^{*} E\left(\theta_{1}, \theta_{2}\right)}{Q_{u}^{2}-\left(n-Q_{u}\right)^{2}} \cdot e^{-i\left(n-Q_{u}\right) \theta_{0}} \\
& +\frac{P_{n}^{*} E^{*}\left(\theta_{1}, \theta_{2}\right)}{Q_{u}^{2}-\left(n+Q_{u}\right)^{2}} \cdot e^{-i\left(n+Q_{u}\right) \theta_{0}},
\end{aligned}
$$

where $E\left(\theta_{1}, \theta_{2}\right)$ and $E^{*}\left(\theta_{1}, \theta_{2}\right)$ are undetermined conjugated functions. Then, by eliminating the secular terms in Eqs. (46) and (47), $E\left(\theta_{1}, \theta_{2}\right)$ and $E^{*}\left(\theta_{1}, \theta_{2}\right)$ can be completely determined as follows:

$$
\begin{aligned}
E\left(\theta_{1}, \theta_{2}\right) & =E\left(\theta_{2}\right)=C_{1} \cdot e^{-i \Lambda_{1} \theta_{2},} \\
E^{*}\left(\theta_{1}, \theta_{2}\right) & =E^{*}\left(\theta_{2}\right)=C_{1}^{*} \cdot e^{i \Lambda_{1} \theta_{2},}
\end{aligned}
$$

where

$$
\Lambda_{1}=\frac{\sum_{n} P_{n} P_{n}^{*}\left[\frac{1}{Q_{u}^{2}-\left(n+Q_{u}\right)^{2}}+\frac{1}{Q_{u}^{2}-\left(n-Q_{u}\right)^{2}}\right]}{2 Q_{u}} .
$$

$C_{1}$ and $C_{1}^{*}$ are conjugated complex numbers that are determined by the initial condition. Finally, by substituting $u_{0}$ and $u_{1}$ into Eq. (43) and letting $\varepsilon=1$, the solution of the original Eq. (37) can be written as

$$
\begin{aligned}
u= & u_{0}+u_{1}+\cdots \\
= & C_{1} \cdot e^{i Q_{r} \theta}+C_{1}^{*} \cdot e^{-i Q_{r} \theta} \\
& -\sum_{n} \frac{P_{n} C_{1}}{Q_{u}^{2}-\left(n+Q_{u}\right)^{2}} \cdot e^{i\left(n+Q_{r}\right) \theta}+\frac{P_{n} C_{1}^{*}}{Q_{u}^{2}-\left(n-Q_{u}\right)^{2}} \cdot e^{i\left(n-Q_{r}\right) \theta}+\frac{P_{n}^{*} C_{1}}{Q_{u}^{2}-\left(n-Q_{u}\right)^{2}} \cdot e^{-i\left(n-Q_{r}\right) \theta} \\
& +\frac{P_{n}^{*} C_{1}^{*}}{Q_{u}^{2}-\left(n+Q_{u}\right)^{2}} \cdot e^{-i\left(n+Q_{r}\right) \theta} .
\end{aligned}
$$

Here,

$$
Q_{r}=-\Lambda_{1}+Q_{u}
$$

is the perturbed radial tune, and $\Lambda_{1}$ is the tune shift provided by the flutter field.

For the vertical oscillation, the solution of the zerothorder approximate equation [Eq. (48)] is

$$
v_{0}=F\left(\theta_{1}, \theta_{2}\right)
$$

The solution of the first-order approximate equation [Eq. (49)] is

$$
v_{1}=\left(\sum_{n} \frac{M_{n}}{n^{2}} \cdot e^{i n \theta_{0}}+\frac{M_{n}^{*}}{n^{2}} \cdot e^{-i n \theta_{0}}\right) \cdot F\left(\theta_{1}, \theta_{2}\right),
$$

where $F\left(\theta_{1}, \theta_{2}\right)$ is the undetermined function. The highorder solutions $v_{2}$ and $v_{3}$ are very small and thus can be neglected (i.e., $v_{2}=v_{3}=0$ ). The undetermined function $F\left(\theta_{1}, \theta_{2}\right)$ can be solved by eliminating the secular terms in Eqs. (50) and (51), which yields

$$
F\left(\theta_{1}, \theta_{2}\right)=C_{2} \cdot e^{i\left(\Lambda_{2} \cdot \theta_{1}+\frac{Q_{v}^{2}}{2 \Lambda_{2}} \cdot \theta_{2}\right)}+C_{2}^{*} \cdot e^{-i\left(\Lambda_{2} \cdot \theta_{1}+\frac{Q_{v}^{2}}{2 \Lambda_{2}} \cdot \theta_{2}\right)} .
$$

Here, $\Lambda_{2}$ can be expressed as

$$
\Lambda_{2}^{2}=2 \sum_{n} \frac{M_{n} M_{n}^{*}}{n^{2}}
$$

$C_{2}$ and $C_{2}^{*}$ are complex conjugated numbers determined by the initial condition. After $F\left(\theta_{1}, \theta_{2}\right)$ is completely determined, the expressions of $v_{0}$ and $v_{1}$ are determined. Finally, the solution of the vertical oscillation [Eq. (39)] follows by letting $\varepsilon=1$ :

$$
\begin{aligned}
v= & v_{0}+v_{1}+\cdots \\
= & \left(C_{2} \cdot e^{i Q_{z} \theta}+C_{2}^{*} \cdot e^{-i Q_{z} \theta}\right) \\
& \cdot\left(1+\sum_{n} \frac{M_{n}}{n^{2}} \cdot e^{i n \theta}+\frac{M_{n}^{*}}{n^{2}} \cdot e^{-i n \theta}\right),
\end{aligned}
$$

where

$$
Q_{z}=\Lambda_{2}+\frac{Q_{v}^{2}}{2 \Lambda_{2}}
$$

is the perturbed vertical tune and $\Lambda_{2}$ is the tune shift provided by the flutter field. Similar to Eqs. (36), (63) is valid only if the flutter field is sufficiently large to satisfy $Q_{z}>0$ (i.e., $\Lambda_{2}>\sqrt{\frac{-Q_{v}^{2}}{2}}$ ) because the derivation is based 
on the assumption that the linear term is a higher-order small quantity than the flutter term.

In order to verify the above formulas, we solved the transverse oscillation equations for the SC200 cyclotron with the fourth-order Runge-Kutta method and above analytical formulas separately. Figures 3 and 4 compare the results of these two methods; the good agreement confirms the accuracy of the analytical formulas. We note that the transverse oscillation is described by $x(\theta)$ and $z(\theta)$. After $u(\theta)$ and $v(\theta)$ are solved, $x(\theta)$ and $z(\theta)$ can be obtained by the relations $x(\theta)=\sqrt{b(\theta)} \cdot u(\theta)$ and $z(\theta)=\sqrt{a(\theta)} \cdot v(\theta)$, which are derived in the Appendix.

\section{B. Transverse oscillation in unstable regions}

\section{1. $2 Q_{r}=2$ resonance}

We consider the radial oscillation given by Eq. (37). The unperturbed radial tune $Q_{u}$ is not equal to the perturbed radial tune $Q_{r}$; in order to consider the effect of the perturbed radial tune, Eq. (37) is transformed to

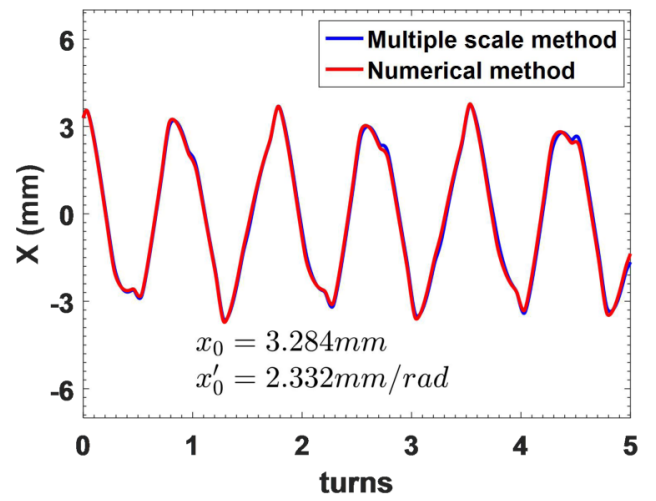

$$
u^{\prime \prime}+Q_{r}^{2} \cdot u=-g(\theta) \cdot u+Q_{r}^{2} \cdot u-Q_{u}^{2} \cdot u
$$

Next, the small parameter $\varepsilon$ is introduced as follows:

$$
u^{\prime \prime}+Q_{r}^{2} \cdot u=-\varepsilon \cdot g(\theta) \cdot u+\varepsilon^{2} \cdot\left(Q_{r}^{2}-Q_{u}^{2}\right) \cdot u .
$$

Then, the corresponding approximate equations are obtained:

$$
\begin{gathered}
D_{0}^{2} u_{0}+Q_{r}^{2} \cdot u_{0}=0, \\
D_{0}^{2} u_{1}+2 D_{0} D_{1} \cdot u_{0}+Q_{r}^{2} \cdot u_{1}=-g(\theta) \cdot u_{0} .
\end{gathered}
$$

The zeroth-order solution is easily obtained with Eq. (66):

$$
u_{0}\left(\theta_{0}, \theta_{1}\right)=E_{1}\left(\theta_{1}\right) \cdot e^{i Q_{r} \cdot \theta_{0}}+E_{1}^{*}\left(\theta_{1}\right) \cdot e^{-i Q_{r} \cdot \theta_{0}} .
$$

By substituting Eq. (68) into Eq. (67), the first-order approximate equation can be written as

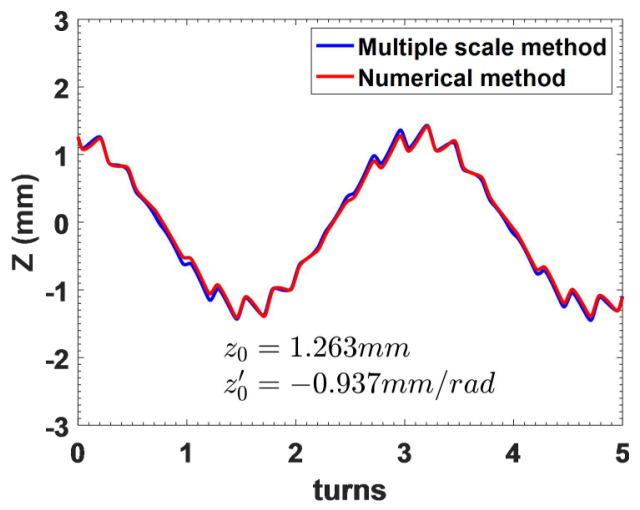

FIG. 3. Transverse oscillations obtained from the numerical method (red) and analytical solutions (blue) for a $100 \mathrm{MeV}$ particle: (left) radial oscillation and (right) vertical oscillation.
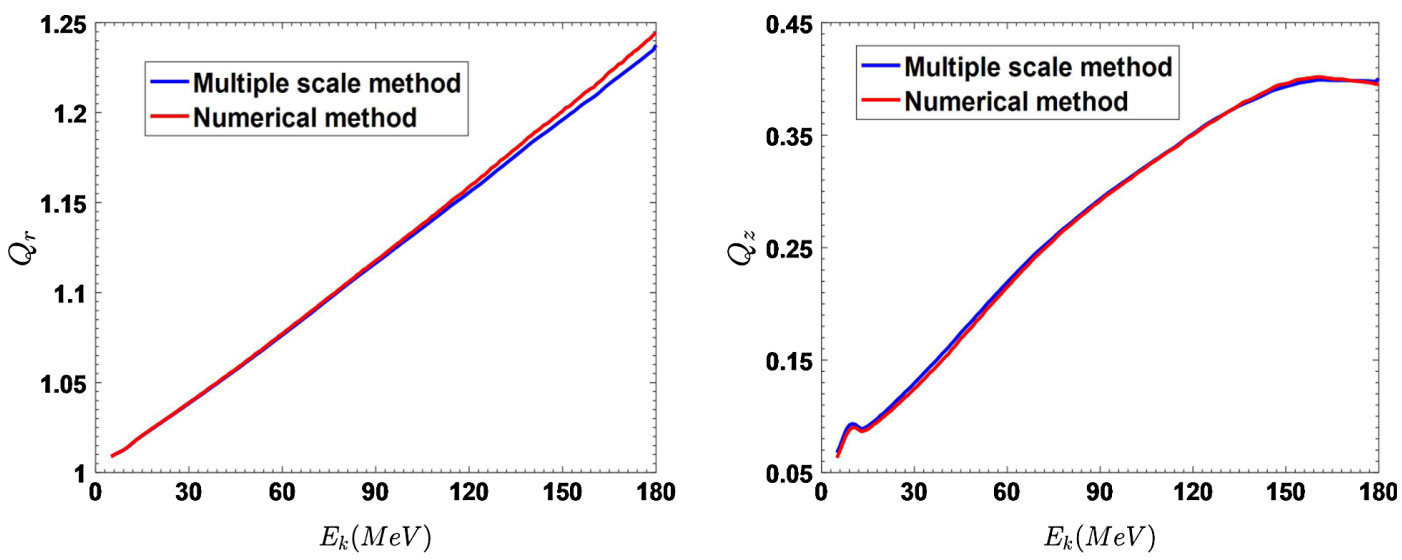

FIG. 4. Betatron tunes from the numerical method (red) and analytical solutions (blue): (left) radial oscillation and (right) vertical oscillation. 
$D_{0}^{2} u_{1}+Q_{r}^{2} \cdot u_{1}=-2\left[i Q_{r} \cdot D_{1} E_{1} \cdot e^{i Q_{r} \theta_{0}}-i Q_{r} \cdot D_{1} E_{1}^{*} \cdot e^{-i Q_{r} \theta_{0}}\right]-\left(\sum_{n} P_{n} e^{i n \theta_{0}}+P_{n}^{*} e^{-i n \theta_{0}}\right) \cdot\left[E_{1} \cdot e^{i Q_{r} \theta_{0}}+E_{1}^{*} \cdot e^{-i Q_{r} \theta_{0}}\right]$.

When the perturbed radial frequency is close to 1 , $Q_{r} \rightarrow 1$; the following term on the right-hand side of Eq. (69) causes additional secular terms:

$$
\begin{gathered}
-2\left[i Q_{r} \cdot D_{1} E_{1} \cdot e^{i Q_{r} \theta_{0}}-i Q_{r} \cdot D_{1} E_{1}^{*} \cdot e^{-i Q_{r} \theta_{0}}\right] \\
-P_{2} E_{1}^{*} \cdot e^{i\left(2-Q_{r}\right) \theta_{0}}-P_{2}^{*} E_{1} \cdot e^{-i\left(2-Q_{r}\right) \theta_{0}} .
\end{gathered}
$$

We introduce the detuning parameter $\sigma_{1}$ to quantify the proximity from the perturbed radial oscillation frequency to 1:

$$
2-Q_{r}=Q_{r}+\varepsilon \cdot \sigma_{1}
$$

By substituting Eq. (71) into Eq. (70) and letting Eq. (70) be zero to eliminate the secular terms, we get

$$
\begin{aligned}
& D_{1} E_{1}\left(\theta_{1}\right)=\frac{i P_{2}}{2 Q_{r}} \cdot e^{i \sigma_{1} \theta_{1}} \cdot E_{1}^{*}\left(\theta_{1}\right), \\
& D_{1} E_{1}^{*}\left(\theta_{1}\right)=-\frac{i P_{2}^{*}}{2 Q_{r}} \cdot e^{-i \sigma_{1} \theta_{1}} \cdot E_{1}\left(\theta_{1}\right) .
\end{aligned}
$$

In order to solve Eq. (72), we introduce the following transformation:

$$
\begin{aligned}
& E_{1}\left(\theta_{1}\right)=W\left(\theta_{1}\right) \cdot e^{i \sigma_{1} \theta_{1} / 2}, \\
& E_{1}^{*}\left(\theta_{1}\right)=W^{*}\left(\theta_{1}\right) \cdot e^{-i \sigma_{1} \theta_{1} / 2} .
\end{aligned}
$$

By separating the real and imaginary parts, Eq. (72) can be transformed into

$$
\frac{d}{d \theta_{1}}\left[\begin{array}{l}
W_{r}\left(\theta_{1}\right) \\
W_{i}\left(\theta_{1}\right)
\end{array}\right]=\left(\begin{array}{cc}
-\frac{P_{2 i}}{2 Q_{r}} & \frac{\sigma_{1}}{2}+\frac{P_{2 r}}{2 Q_{r}} \\
-\frac{\sigma_{1}}{2}+\frac{P_{2 r}}{2 Q_{r}} & \frac{P_{2 i}}{2 Q_{r}}
\end{array}\right)\left[\begin{array}{c}
W_{r}\left(\theta_{1}\right) \\
W_{i}\left(\theta_{1}\right)
\end{array}\right],
$$

where $W(\theta)=W_{r}(\theta)+i \cdot W_{i}(\theta)$ and $P_{2}=P_{2 r}+i \cdot P_{2 i}$. The characteristic equation of the above linear differential equations can be written as

$$
\eta^{2}-\frac{\left|P_{2}\right|^{2}}{\left(2 Q_{r}\right)^{2}}+\left(\frac{\sigma_{1}}{2}\right)^{2}=0
$$

where $\eta$ is the eigenvalue. When $\eta^{2}<0$ (i.e., $\sigma_{1}^{2}>\frac{\left|P_{2}\right|^{2}}{Q_{r}^{2}}$ ), Eq. (74) has trigonometric form solutions. In this case,
$W_{r}\left(\theta_{1}\right)$ and $W_{i}\left(\theta_{1}\right)$ are bounded, and the system is stable. When $\eta^{2}>0$ (i.e., $\sigma_{1}^{2}<\frac{\left|P_{2}\right|^{2}}{Q_{r}^{2}}$ ), Eq. (74) has exponent form solutions:

$$
\begin{aligned}
& W_{r}\left(\theta_{1}\right)=C_{3} \cdot e^{\eta \theta_{1}}+C_{4} \cdot e^{-\eta \theta_{1}}, \\
& W_{i}\left(\theta_{1}\right)=\frac{\eta+\frac{P_{2 i}}{2 Q_{r}}}{\frac{\sigma_{1}}{2}+\frac{P_{2 r}}{2 Q_{r}}} C_{3} \cdot e^{\eta \theta_{1}}+\frac{-\eta+\frac{P_{2 i}}{2 Q_{r}}}{\frac{\sigma_{1}}{2}+\frac{P_{2 r}}{2 Q_{r}}} C_{4} \cdot e^{-\eta \theta_{1}},
\end{aligned}
$$

where $C_{3}$ and $C_{4}$ are constants determined by the initial condition. In this case, $W_{r}\left(\theta_{1}\right)$ and $W_{i}\left(\theta_{1}\right)$ are unbounded, and the system is unstable. Thus, the stability condition for the radial oscillation near $2 Q_{r}=2$ is $\left|\sigma_{1}\right|>\left|\frac{P_{2}}{Q_{r}}\right|$; that is, the radial oscillation near $2 Q_{r}=2$ is stable only when the distance to $2 Q_{r}=2$ satisfies $\left|2-2 Q_{r}\right|>\varepsilon\left|\frac{P_{2}}{Q_{r}}\right|$. Otherwise, the amplitude continuously increases, and the system is unstable. After $W(\theta)$ is solved, the coefficients $E_{1}\left(\theta_{1}\right)$ and $E_{1}^{*}\left(\theta_{1}\right)$ can be completely determined from Eq. (73). Then, the first-order solution can be expressed as

$$
\begin{aligned}
u_{1}= & -\sum_{n \neq 2} \frac{P_{n} E_{1}}{Q_{r}^{2}-\left(n+Q_{r}\right)^{2}} \cdot e^{i\left(n+Q_{r}\right) \theta_{0}} \\
& +\frac{P_{n} E_{1}^{*}}{Q_{r}^{2}-\left(n-Q_{r}\right)^{2}} \cdot e^{i\left(n-Q_{r}\right) \theta_{0}}+\frac{P_{n}^{*} E_{1}}{Q_{r}^{2}-\left(n-Q_{r}\right)^{2}} \\
& \cdot e^{-i\left(n-Q_{r}\right) \theta_{0}}+\frac{P_{n}^{*} E_{1}^{*}}{Q_{r}^{2}-\left(n+Q_{r}\right)^{2}} \cdot e^{-i\left(n+Q_{r}\right) \theta_{0}} .
\end{aligned}
$$

Finally, by letting $\varepsilon=1$ in Eq. (43), a solution of Eq. (37) near $2 Q_{r}=2$ follows. The details are not repeated here.

According to Eq. (68), the amplitude of the transverse oscillation can be written as follows:

$$
A_{m}(\theta)=2 \sqrt{E_{1}(\theta) \cdot E_{1}^{*}(\theta)} .
$$

The increasing rate of the amplitude is proportional to $e^{\eta \theta}$; for every turn a particle rotates, its amplitude increases approximately $e^{2 \pi \eta}$ times. The driving term of the $2 Q_{r}=2$ resonance is the second harmonic; a larger second harmonic value will cause a faster increasing rate in the amplitude growth. The left side of Fig. 5 shows the relations between the perturbed radial tune $Q_{r}$ and increasing rate $e^{2 \pi \eta}$ for different second harmonic values; we can also use this to find the resonance width near $2 Q_{r}=2$. The right side of Fig. 5 shows the transverse oscillation obtained from the numerical integration and analytical formulas in 

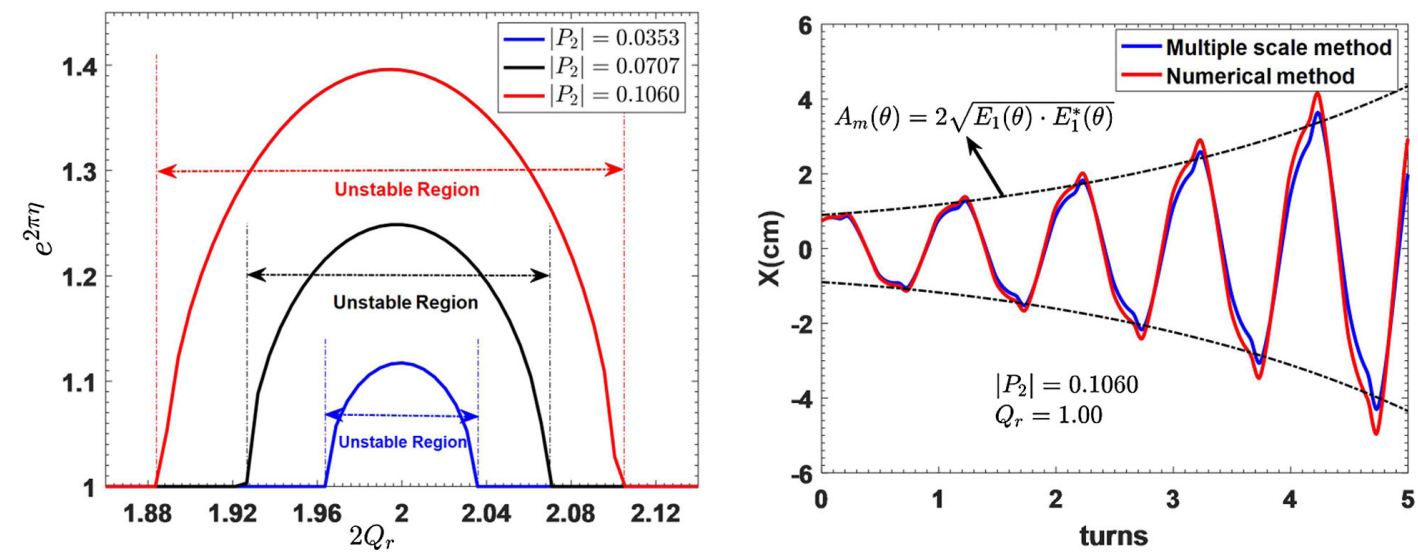

FIG. 5. (Left) Relationship between the perturbed radial tune $Q_{r}$ and increasing rate of the amplitude $e^{2 \pi \eta}$ for different second harmonic values. (Right) Radial oscillations obtained by the numerical simulation and analytical solution.

the vicinity of $2 Q_{r}=2$. The difference is very small, and the amplitude variation coincides very well with the description of Eq. (78).

\section{2. $Q_{z}=1 / 2$ resonance}

We consider the vertical oscillation equation given by Eq. (38). The small parameter $\varepsilon$ can be introduced as follows:

$$
\begin{aligned}
v^{\prime \prime}+Q_{z}^{2} \cdot v= & -\varepsilon \cdot h(\theta) \cdot v+\varepsilon \cdot J(\theta) \cdot u v \\
& +\varepsilon^{2} \cdot\left(Q_{z}^{2}-Q_{v}^{2}\right) \cdot v .
\end{aligned}
$$

Two scales are considered: $\theta_{0}=\theta$ and $\theta_{1}=\varepsilon \cdot \theta$. The corresponding approximate equations are

$$
D_{0}^{2} v_{0}+Q_{z}^{2} \cdot v_{0}=0
$$

$$
D_{0}^{2} v_{1}+Q_{z}^{2} \cdot v_{1}+2 D_{0} D_{1} \cdot v_{0}=-h(\theta) \cdot v_{0}+J(\theta) \cdot u_{0} v_{0} .
$$

The zeroth-order approximation can be written as

$$
v_{0}\left(\theta_{0}, \theta_{1}\right)=F_{1}\left(\theta_{1}\right) \cdot e^{i Q_{z} \theta_{0}}+F_{1}^{*}\left(\theta_{1}\right) \cdot e^{-i Q_{z} \theta_{0}},
$$

where $F_{1}\left(\theta_{1}\right)$ and $F_{1}^{*}\left(\theta_{1}\right)$ are undetermined conjugated complex functions. By substituting Eq. (82) into Eq. (81), $-h(\theta) \cdot v_{0}$ and $J(\theta) \cdot u_{0} v_{0}$ are written as

$$
\begin{aligned}
-h(\theta) \cdot v_{0} & =-\sum_{n} M_{n} F_{1} \cdot e^{i\left(n+Q_{z}\right) \theta_{0}}+M_{n}^{*} F_{1} \cdot e^{-i\left(n-Q_{z}\right) \theta_{0}}+M_{n} F_{1}^{*} \cdot e^{i\left(n-Q_{z}\right) \theta_{0}}+M_{n}^{*} F_{1}^{*} \cdot e^{-i\left(n+Q_{z}\right) \theta_{0}}, \\
J(\theta) u_{0} v_{0}= & R_{0}\left[E_{1} F_{1} \cdot e^{i\left(Q_{r}+Q_{z}\right) \theta_{0}}+E_{1} F_{1}^{*} \cdot e^{i\left(Q_{r}-Q_{z}\right) \theta_{0}}+E_{1}^{*} F_{1} \cdot e^{-i\left(Q_{r}-Q_{z}\right) \theta_{0}}+E_{1}^{*} F_{1}^{*} \cdot e^{-i\left(Q_{r}+Q_{z}\right) \theta_{0}}\right] \\
& +\sum_{n} R_{n} \cdot E_{1} F_{1} \cdot e^{i\left(Q_{r}+Q_{z}+n\right) \theta_{0}}+R_{n} \cdot E_{1} F_{1}^{*} \cdot e^{i\left(Q_{r}-Q_{z}+n\right) \theta_{0}}+R_{n} \cdot E_{1}^{*} F_{1} \cdot e^{-i\left(Q_{r}-Q_{z}-n\right) \theta_{0}} \\
& +R_{n} \cdot E_{1}^{*} F_{1}^{*} \cdot e^{-i\left(Q_{r}+Q_{z}-n\right) \theta_{0}}+R_{n}^{*} \cdot E_{1} F_{1} \cdot e^{i\left(Q_{r}+Q_{z}-n\right) \theta_{0}}+R_{n}^{*} \cdot E_{1} F_{1}^{*} \cdot e^{i\left(Q_{r}-Q_{z}-n\right) \theta_{0}} \\
& +R_{n}^{*} \cdot E_{1}^{*} F_{1} \cdot e^{-i\left(Q_{r}-Q_{z}+n\right) \theta_{0}}+R_{n}^{*} \cdot E_{1}^{*} F_{1}^{*} \cdot e^{-i\left(Q_{r}+Q_{z}+n\right) \theta_{0}} .
\end{aligned}
$$

As shown above, two cases need to be considered.

Case 1.-When $Q_{z} \rightarrow \frac{1}{2}$, then $1-Q_{z} \rightarrow Q_{z}$. The term $M_{1}^{*} F_{1} \cdot e^{-i\left(1-Q_{z}\right) \theta_{0}}+M_{1} F_{1}^{*} \cdot e^{i\left(1-Q_{z}\right) \theta_{0}}$ in Eq. (83) causes additional secular terms.

Case 2.-When $Q_{r}-2 Q_{z} \rightarrow 1$, then $Q_{r}-Q_{z}-1 \rightarrow Q_{z}$. The term $R_{1} \cdot E^{*} F_{1} \cdot e^{-i\left(Q_{r}-Q_{z}-1\right) \theta_{0}}+R_{1}^{*} \cdot E F_{1}^{*} \cdot e^{i\left(Q_{r}-Q_{z}-1\right) \theta_{0}}$ in Eq. (84) causes additional secular terms.

For case 1 , the detuning parameter $\sigma_{2}$ is introduced to quantitatively describe the nearness of the perturbed vertical tune to $\frac{1}{2}$ :

$$
\frac{1}{2}-Q_{z}=\varepsilon \cdot \frac{\sigma_{2}}{2}
$$

Substituting Eq. (85) into Eq. (83) and eliminating the secular terms that occur in Eq. (81) yields the condition for determining $F_{1}\left(\theta_{1}\right)$ and $F_{1}^{*}\left(\theta_{1}\right)$ as well as the stable condition

$$
\left|\sigma_{2}\right|>\left|\frac{M_{1}}{2 Q_{z}}\right|
$$


If $\left|\sigma_{2}\right|<\left|\frac{M_{1}}{2 Q_{z}}\right|$, then the system is unstable. In this case, the unbounded solutions of $F_{1}\left(\theta_{1}\right)$ and $F_{1}^{*}\left(\theta_{1}\right)$ are obtained as

$F_{1}\left(\theta_{1}\right)=V_{1}\left(\theta_{1}\right) \cdot e^{\frac{i \sigma_{2} \theta_{1}}{2}}, \quad F_{1}^{*}\left(\theta_{1}\right)=V_{1}^{*}\left(\theta_{1}\right) \cdot e^{-\frac{i \sigma_{2} \theta_{1}}{2}}$,

where

$$
\begin{aligned}
& V_{1 r}\left(\theta_{1}\right)=C_{5} \cdot e^{\eta \theta_{1}}+C_{6} \cdot e^{-\eta \theta_{1}}, \\
& V_{1 i}\left(\theta_{1}\right)=\frac{\eta+\frac{M_{1 i}}{2 Q_{z}}}{\frac{\sigma_{2}}{2}+\frac{M_{1 r}}{2 Q_{z}}} C_{5} \cdot e^{\eta \theta_{1}}+\frac{-\eta+\frac{M_{1 i}}{2 Q_{z}}}{\frac{\sigma_{2}}{2}+\frac{M_{1 r}}{2 Q_{z}}} C_{6} \cdot e^{-\eta \theta_{1}} .
\end{aligned}
$$

Here, $V_{1}\left(\theta_{1}\right)=V_{1 r}\left(\theta_{1}\right)+i \cdot V_{1 i}\left(\theta_{1}\right), C_{5}$ and $C_{6}$ are real numbers determined by the initial condition, $M_{1 i}$ and $M_{1 r}$ are the real and imaginary parts, respectively, of $M_{1}$. With the above procedure, the undetermined functions $F_{1}\left(\theta_{1}\right)$ and $F_{1}^{*}\left(\theta_{1}\right)$ are completely determined. Then, $v_{0}\left(\theta_{0}, \theta_{1}\right)$ and $v_{1}\left(\theta_{0}, \theta_{1}\right)$ can be determined. Finally, the solutions of Eq. (38) follow by letting $\varepsilon=1$. The driving terms of $Q_{z}=\frac{1}{2}$ resonance is the first harmonic. In Fig. 6, the left figure shows the unstable regions and resonance width near $Q_{z}=\frac{1}{2}$ for different first harmonic values. The right figure is the comparison of the vertical motion obtained from the numerical simulation and the analytical formulas under the same initial condition; the difference is very small, which proves the analytical formulas to be accurate.

\section{3. $Q_{r}-2 Q_{z}=1$ resonance}

For case 2, a small parameter $\sigma_{3}$ is introduced to quantitatively describe the proximity of $Q_{r}-2 Q_{z}$ to 1 :

$$
Q_{r}-2 Q_{z}-1=\varepsilon \cdot \sigma_{3} \text {. }
$$

Then, all terms that cause secular terms in Eq. (81) are written as

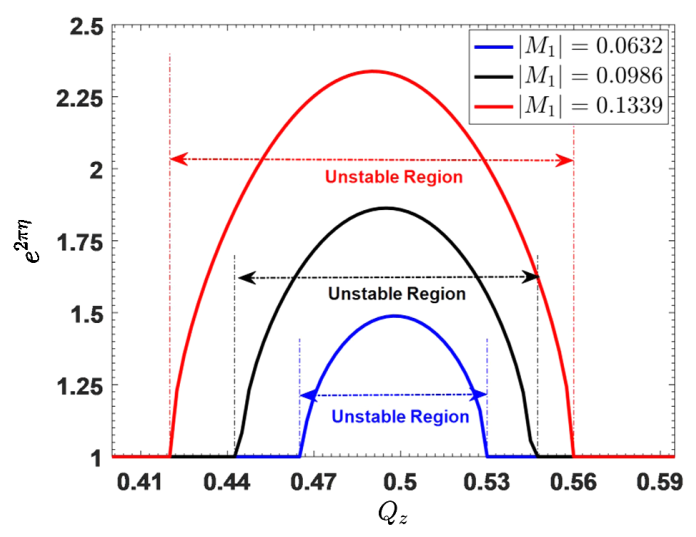

$$
\begin{aligned}
& -2 i Q_{z}\left[\frac{\partial F_{1}\left(\theta_{1}\right)}{\partial \theta_{1}} \cdot e^{i Q_{z} \theta_{0}}-\frac{\partial F_{1}^{*}\left(\theta_{1}\right)}{\partial \theta_{1}} \cdot e^{-i Q_{z} \theta_{0}}\right] \\
& \quad+\left[R_{1}^{*} \cdot E F_{1}^{*} \cdot e^{i \sigma_{3} \theta_{1}} \cdot e^{i Q_{z} \theta_{0}}+R_{1} \cdot E^{*} F_{1} \cdot e^{-i \sigma_{3} \theta_{1}} \cdot e^{-i Q_{z} \theta_{0}}\right] .
\end{aligned}
$$

We let Eq. (90) be zero to eliminate the secular terms, which results in

$$
\begin{gathered}
2 \frac{\partial F_{1}}{\partial \theta_{1}} \cdot\left(i Q_{z}\right) \cdot e^{i Q_{z} \theta_{0}}=R_{1}^{*} \cdot E F_{1}^{*} \cdot e^{i Q_{z} \theta_{0}} \cdot e^{i \sigma_{3} \theta_{1}}, \\
2 \frac{\partial F_{1}^{*}}{\partial \theta_{1}} \cdot\left(-i Q_{z}\right) \cdot e^{-i Q_{z} \theta_{0}}=R_{1} \cdot E^{*} F_{1} \cdot e^{-i Q_{z} \theta_{0}} \cdot e^{-i \sigma_{3} \theta_{1}},
\end{gathered}
$$

where $E=E\left(\theta_{2}\right)$ and $E^{*}=E^{*}\left(\theta_{2}\right)$. The specific expressions of $E$ and $E^{*}$ are given in Eq. (54). Because $\theta_{2}$ varies very slowly compared to $\theta_{0}$ and $\theta_{1}, E\left(\theta_{2}\right)$ and $E^{*}\left(\theta_{2}\right)$ can be treated as constants:

$$
\begin{aligned}
E\left(\theta_{2}\right) & \approx C_{1}=C_{1 r}+i \cdot C_{1 i}, \\
E^{*}\left(\theta_{2}\right) \approx C_{1}^{*} & =C_{1 r}-i \cdot C_{1 i} .
\end{aligned}
$$

Here, $C_{1 r}$ and $C_{1 i}$ are real numbers that are directly related to the initial radial amplitude. Similar to the procedure described for Eqs. (73)-(75), Eq. (91) can be transformed into linear differential equations by introducing a variable transformation, then the stable condition of the system is obtained by solving the characteristic equation, which gives

$$
\left|\sigma_{3}\right|>\left|\frac{R_{1} \cdot C_{1}}{2 Q_{z}}\right|
$$

When $\left|\sigma_{3}\right|<\left|\frac{R_{1} \cdot C_{1}}{2 Q_{z}}\right|$, the system is unstable; then, Eq. (91) has an exponential form solution:

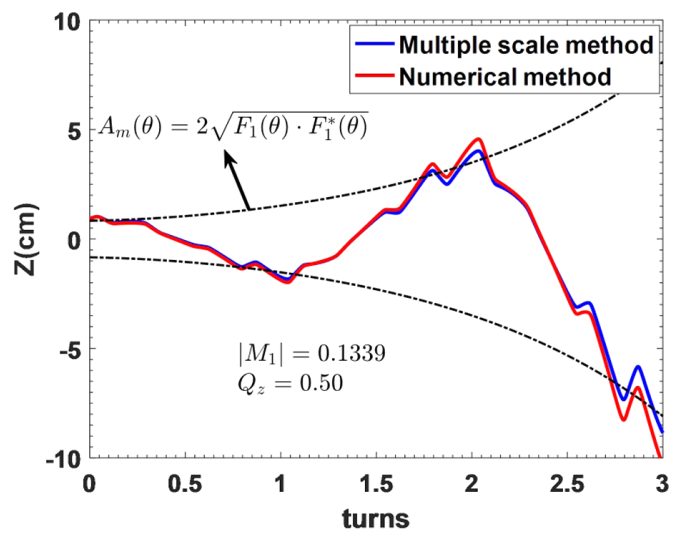

FIG. 6. (Left) Relationship between the perturbed vertical tune $Q_{z}$ and increasing rate $e^{2 \pi \eta}$ for different first harmonic values near $Q_{z}=\frac{1}{2}$. (Right) Vertical oscillations obtained by the numerical simulation and analytical solution. 

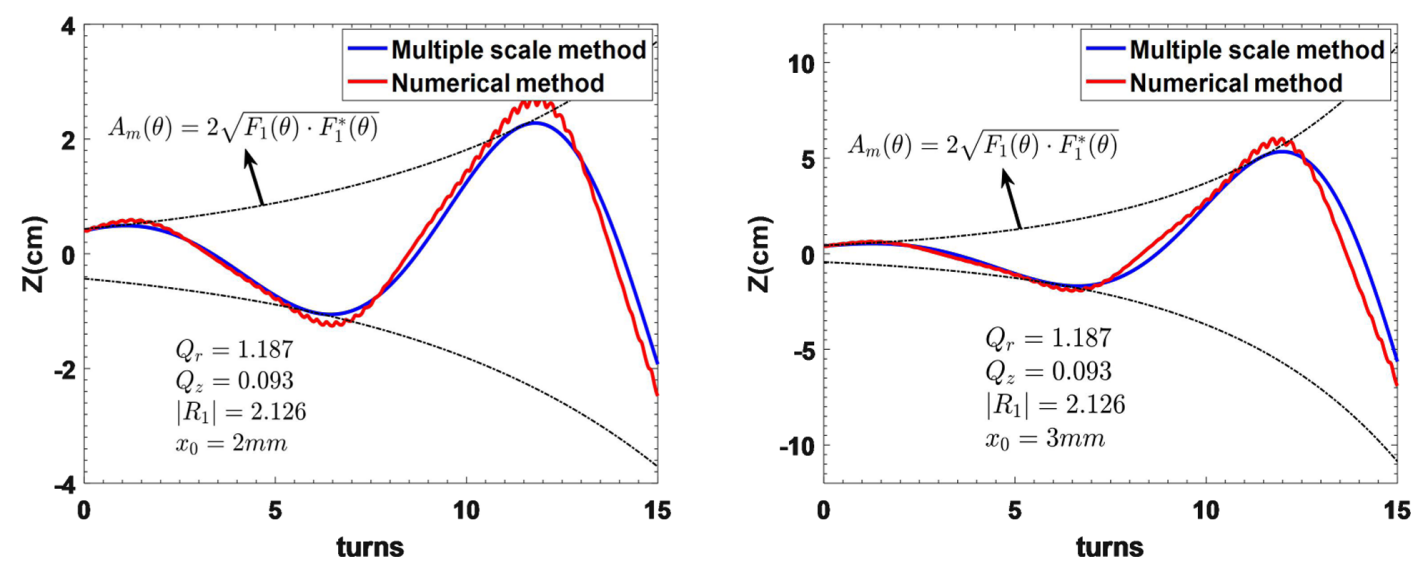

FIG. 7. Vertical oscillations obtained by the numerical method (red) and analytical formulas (blue) near $Q_{r}-2 Q_{z}=1$ with the same vertical amplitude at initial radial amplitudes of (left) $2 \mathrm{~mm}$ and (right) $3 \mathrm{~mm}$.

$$
F_{1}\left(\theta_{1}\right)=V_{2}\left(\theta_{1}\right) \cdot e^{\frac{i \sigma_{3} \cdot \theta_{1}}{2}}
$$

where

$$
\begin{aligned}
V_{2 r}= & C_{7} \cdot e^{\eta \theta_{1}}+C_{8} \cdot e^{-\eta \theta_{1}}, \\
V_{2 i}= & \frac{\eta-\frac{R_{r} C_{1 i}-R_{i} C_{1 r}}{2 Q_{z}}}{\frac{\sigma_{3}}{2}-\frac{R_{r} C_{1 r}+R_{i} C_{1 i}}{2 Q_{z}}} \cdot C_{7} \cdot e^{\eta \theta_{1}} \\
& +\frac{-\eta-\frac{R_{r} C_{1 i}-R_{i} C_{1 r}}{2 Q_{z}}}{\frac{\sigma_{3}}{2}-\frac{R_{r} C_{1 r}+Q_{i} C_{1 i}}{2 Q_{z}}} \cdot C_{8} \cdot e^{-\eta \theta_{1}} .
\end{aligned}
$$

Here, $V_{2 r}$ and $V_{2 i}$ are real and imaginary parts of $V_{2} . C_{7}$ and $C_{8}$ are constants determined by the initial condition. Finally, the solutions of Eq. (38) near $Q_{r}-2 Q_{z}=1$ can be obtained.

As presented above, the dynamic behavior of the vertical oscillation near $Q_{r}-2 Q_{z}=1$ is related to not only the values of $Q_{r}$ and $Q_{z}$ and its driving term $\left|R_{1}\right|$ but also to the initial radial amplitude $\left|C_{1}\right|$. Figure 7 compares the vertical oscillation of particles with initial radial amplitudes of 2 and $3 \mathrm{~mm}$ (the initial vertical amplitudes are the same). Particles with a larger initial radial amplitude have a faster increase in the vertical amplitude.

\section{CONCLUSION}

We modified the MMS to obtain approximate analytical solutions of the Mathieu equation in stable and unstable regions for both $\delta \geq 0$ and $\delta<0$. Numerical simulations were carried out to investigate the dynamic performance of the Mathieu equation. Very good agreement was obtained between the results of the numerical integration and analytical solutions, which means that the modified MMS is useful for obtaining the analytical solution of the Mathieu equation.

The modified MMS was applied to the nonlinear transverse oscillation equations of a cyclotron. The equations of the transverse oscillation were derived and transformed into the Mathieu equation. Analytical solutions were obtained for the transverse oscillation in the stable and unstable regions (i.e., vicinity of the resonances $2 Q_{r}=2$, $Q_{z}=1 / 2$, and $Q_{r}-2 Q_{z}=1$ ). The validity of the analytical solutions was confirmed by a comparison with the direct numerical integration results. Useful results such as the analytical solution of the transverse oscillation frequency, increasing rate of the amplitude in the unstable regions, and resonance width were also derived, and the driving terms and stability conditions were obtained from the analytical results, which can help improve the understanding of the dynamics and provide a reference for the design of a magnetic field for a cyclotron.

In this paper, we considered an ideal case without magnetic imperfections. In other words, we assumed that only the $B z$ component is nonzero in the middle plane. Future studies could continue to explore this issue by considering field imperfections. For example, the asymmetry of magnetic sectors may lead to a $\mathrm{Br}$ component in the middle plane. More coupling resonances can be studied by taking into account the $\mathrm{Br}$ component. A more general and practical situation could also be evaluated by considering the electric field.

\section{ACKNOWLEDGMENTS}

This work was supported by: (1) National Natural Science Foundation of China under Grants No. 11775258 and No. 11575237; (2) International Scientific and Technological Cooperation Project of Anhui (Grant No. 1704e1002207).

\section{APPENDIX: DERIVATION OF THE TRANSVERSE OSCILLATION EQUATIONS}

The motion of particles in electromagnetic fields is described by the well-known Lorentz force formula: 


$$
\vec{F}=q \vec{v} \times \vec{B}+q \vec{E} .
$$

In this study, the electric field was not considered. If the azimuth $\theta$ is chosen as the independent variable and Eq. (A1) is decomposed in a cylindrical coordinate system, the equations of motion for charged particles in a static magnetic field are obtained as follows:

$$
\begin{aligned}
r^{\prime \prime}-\frac{2 r^{\prime 2}}{r}-r= & -\frac{q}{P}\left(1+\frac{r^{\prime 2}}{r^{2}}+\frac{z^{\prime 2}}{r^{2}}\right)^{\frac{1}{2}}\left[\left(r^{2}+r^{\prime 2}\right) B_{z}\right. \\
& \left.-r^{\prime} z^{\prime} B_{r}-r z^{\prime} B_{\theta}\right], \\
z^{\prime \prime}-\frac{2 r^{\prime} z^{\prime}}{r}= & \frac{q}{P}\left(1+\frac{r^{\prime 2}}{r^{2}}+\frac{z^{\prime 2}}{r^{2}}\right)^{\frac{1}{2}}\left[\left(r^{2}+z^{\prime 2}\right) B_{r}\right. \\
& \left.-r^{\prime} z^{\prime} B_{z}-r r^{\prime} B_{\theta}\right],
\end{aligned}
$$

where $P$ is the total momentum of a particle; $r=r(\theta)$ and $z=z(\theta)$ are the radial and vertical coordinates, respectively, describing the particle's motion; the superscript represents the derivation with respect to the independent variable; and $B_{r}=B_{r}(r, \theta, z), B_{\theta}=B_{\theta}(r, \theta, z)$, and $B_{z}=$ $B_{z}(r, \theta, z)$ are the radial, azimuth, and vertical components, respectively, of $\vec{B}$.

Cyclotron magnets have median plane symmetry. Hence, only $B_{z}$ is nonzero at $z=0$. Once the $2 \mathrm{D}$ numerical field map of the vertical component in the middle plane $\left.B_{z}(r, \theta, z)\right|_{z=0}$ is given, the equilibrium orbit (i.e., closed orbit in the middle plane) $r_{e}(\theta)$ can be determined with Gordon's analytical formula [27] or the numerical procedure [28]. The magnetic field and equilibrium orbits for the SC200 cyclotron are shown in Figs. 8 and 9.

The transverse oscillation is described by $x(\theta)$ and $z(\theta)$. These are defined as

$$
x(\theta)=r(\theta)-r_{e}(\theta), \quad z(\theta)=z(\theta)-0,
$$

where $x(\theta)$ and $z(\theta)$ are the radial and vertical displacements, respectively, in relation to the equilibrium orbit. Because the displacements $x(\theta)$ and $z(\theta)$ are usually small, all of the terms in Eqs. (A2) and (A3) can be expanded in a Taylor series around the equilibrium orbit. Then, transverse oscillation equations that are accurate to the second order are obtained as follows:

$$
\begin{aligned}
x^{\prime \prime}+ & A(\theta) x^{\prime}+B(\theta) x \\
= & C_{1}(\theta) \cdot x^{2}+C_{2}(\theta) \cdot x^{\prime 2}+C_{3}(\theta) \cdot x x^{\prime} \\
& +C_{4}(\theta) \cdot z z^{\prime}+C_{5}(\theta) \cdot z^{\prime 2}, \\
z^{\prime \prime}+ & \alpha(\theta) z^{\prime}+\beta(\theta) z \\
= & D_{1}(\theta) \cdot x z+D_{2}(\theta) \cdot x z^{\prime}+D_{3}(\theta) \cdot x^{\prime} z \\
& +D_{4}(\theta) \cdot x^{\prime} z^{\prime} .
\end{aligned}
$$

Coefficients such as $A(\theta)$ and $B(\theta)$ are functions of the equilibrium orbit parameters, which have the same period $T=2 \pi$ as the equilibrium orbit.

For a compact isochronous cyclotron such as the SC200, all second-order terms in the radial equation such as $C_{1}(\theta) \cdot x^{2}$ and $C_{2}(\theta) \cdot x^{\prime 2}$ have little effect on a particle's motion. All second-order terms in the vertical equation except for $D_{1}(\theta) \cdot x z$ also have little effect on the particle's motion. This is because $x^{\prime}$ and $z^{\prime}$ are smaller than $x$ and $z$ by an order of magnitude or more for the SC200 cyclotron; $x^{\prime}$ and $z^{\prime}$ are approximately $0 \sim 10^{-3} \mathrm{~m} / \mathrm{rad}$, while $x$ and $z$ are approximately $0-10^{-2} \mathrm{~m}$. Consequently, the second-order terms with the exceptions of $D_{1}(\theta) \cdot x z$ and $C_{1}(\theta) \cdot x^{2}$ can be treated as high-order terms. Moreover, the mean value of $B(\theta)$ is larger than that of $\beta(\theta)$ by an order of magnitude or more, which indicates that the linear part of the radial oscillation $B(\theta) \cdot x$ is strong and its response to the nonlinear terms is weak. Meanwhile, the linear part of the vertical oscillation $\beta(\theta) \cdot z$ is weaker and more susceptible to the influence of the nonlinear term. By ignoring all terms on the right side of Eqs. (A5) and (A6) except for $D_{1}(\theta) \cdot x z$, the transverse oscillation equations can be simplified as follows:

$$
\begin{gathered}
x^{\prime \prime}+A(\theta) x^{\prime}+B(\theta) x=0, \\
z^{\prime \prime}+\alpha(\theta) z^{\prime}+\beta(\theta) z=D_{1}(\theta) \cdot x z
\end{gathered}
$$

Equations (A7) and (A8) are Hill-type differential equations and can be further simplified by introducing two parameters:

$$
a(\theta)=\sqrt{r_{e}^{2}+r_{e}^{\prime 2}}, \quad b(\theta)=\frac{\left(r_{e}^{2}+r_{e}^{\prime 2}\right)^{\frac{3}{2}}}{r_{e}^{2}} .
$$

Then, Eqs. (A5) and (A6) can be written as

$$
\begin{gathered}
x^{\prime \prime}-\frac{b^{\prime}(\theta)}{b(\theta)} x^{\prime}+B(\theta) x=0, \\
z^{\prime \prime}-\frac{a^{\prime}(\theta)}{a(\theta)} z^{\prime}+\beta(\theta) z=D_{1}(\theta) \cdot x z .
\end{gathered}
$$

The following transformation can be applied:

$$
x=\sqrt{b(\theta)} \cdot u, \quad z=\sqrt{a(\theta)} \cdot v .
$$

Then, Eqs. (A10) and (A11) can be further simplified as

$$
\begin{gathered}
u^{\prime \prime}+G(\theta) \cdot u=0, \\
v^{\prime \prime}+H(\theta) \cdot v=J(\theta) \cdot u v,
\end{gathered}
$$



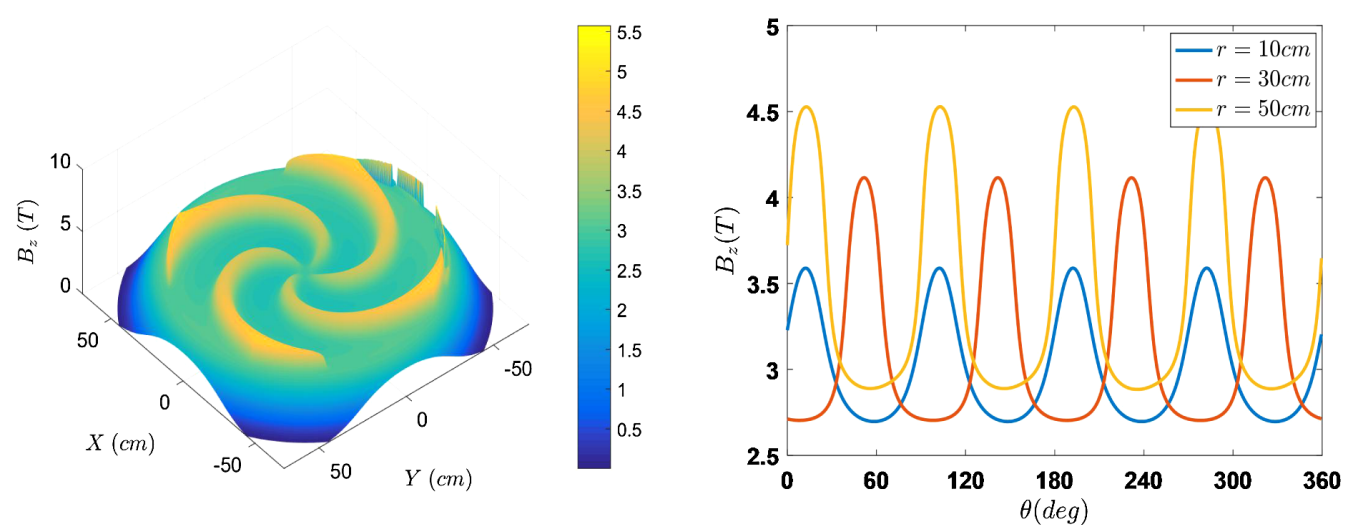

FIG. 8. (Left) 2D magnetic field map in the middle plane for the SC200 cyclotron. (Right) Magnetic field with respect to the azimuth for different radii.
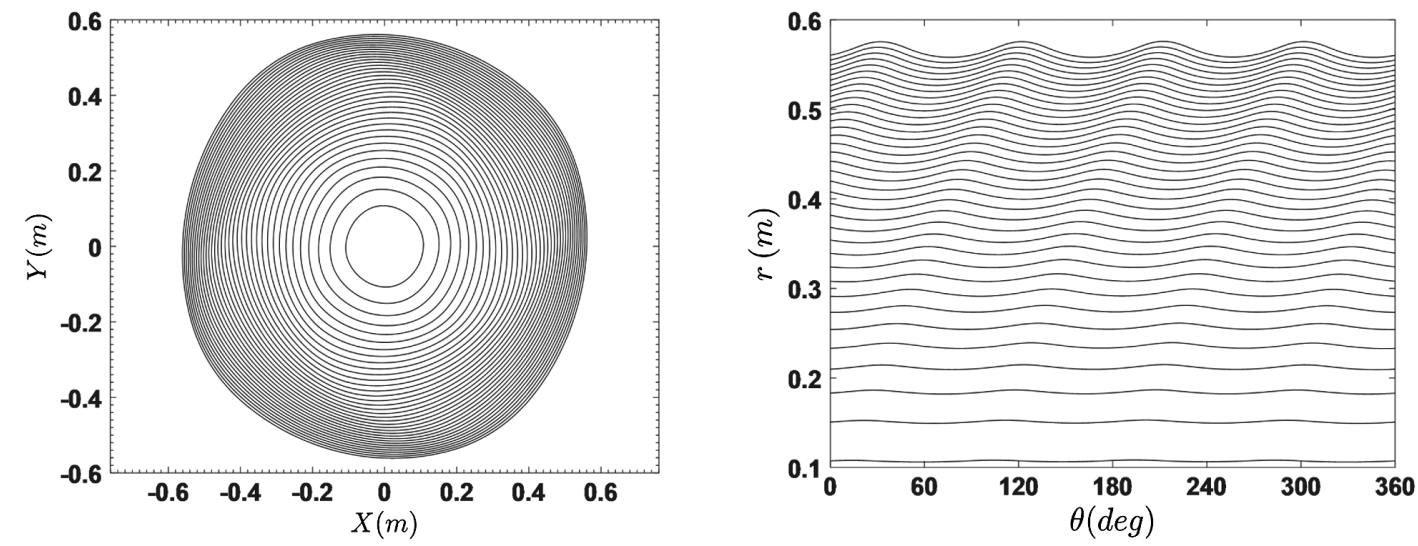

FIG. 9. Equilibrium orbits for the SC200 cyclotron.

where $G(\theta)=\frac{b^{\prime \prime}}{b}-\frac{3}{4}\left(\frac{b^{\prime}}{b}\right)^{2}+B(\theta), H(\theta)=\frac{a^{\prime \prime}}{a}-\frac{3}{4}\left(\frac{a^{\prime}}{a}\right)^{2}+\beta(\theta)$, and $J(\theta)=\sqrt{b(\theta)} \cdot D_{1}(\theta)$. These three coefficients are periodic and have the same period $T=2 \pi$ as the equilibrium orbit. Expanding the coefficients into Fourier series gives

$$
G(\theta)=Q_{u}^{2}+\sum_{n} P_{n} e^{i n \theta}+P_{n}^{*} e^{-i n \theta},
$$

$$
\begin{gathered}
H(\theta)=Q_{v}^{2}+\sum_{n} M_{n} e^{i n \theta}+M_{n}^{*} e^{-i n \theta}, \\
J(\theta)=R_{0}+\sum_{n} R_{n} e^{i n \theta}+R_{n}^{*} e^{-i n \theta} .
\end{gathered}
$$

For simplification, we let $g(\theta)=\sum_{n} P_{n} e^{i n \theta}+P_{n}^{*} e^{-i n \theta}$ and $h(\theta)=\sum_{n} M_{n} e^{i n \theta}+M_{n}^{*} e^{-i n \theta}$. Then, Eqs. (A13) and (A14) become
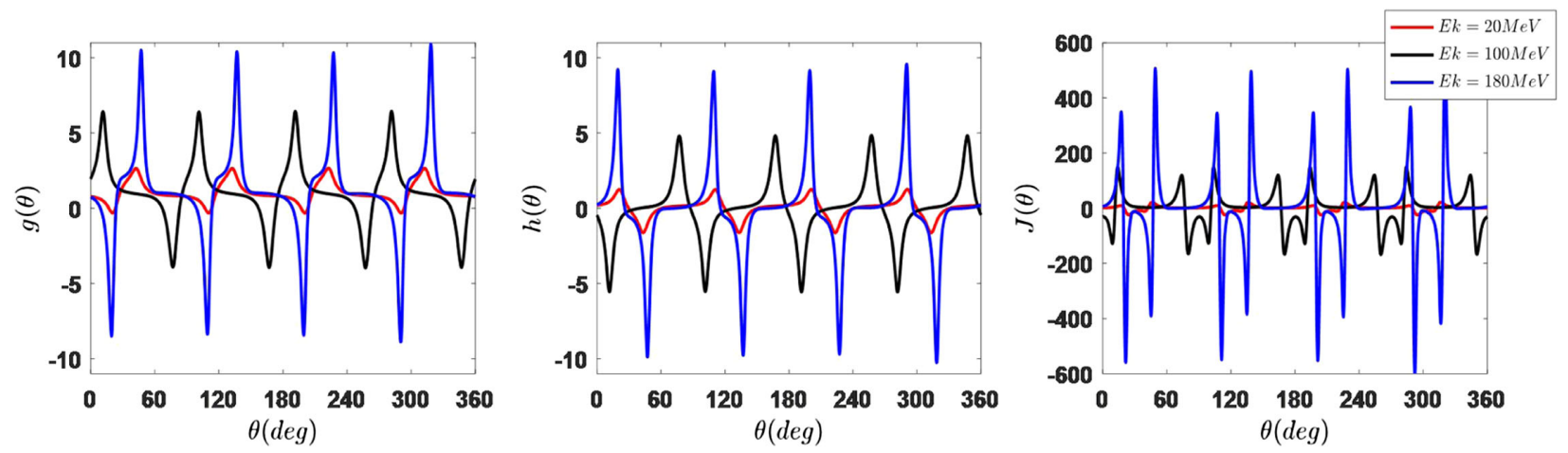

FIG. 10. Coefficients $g(\theta), h(\theta)$, and $J(\theta)$ for different energy particles. 

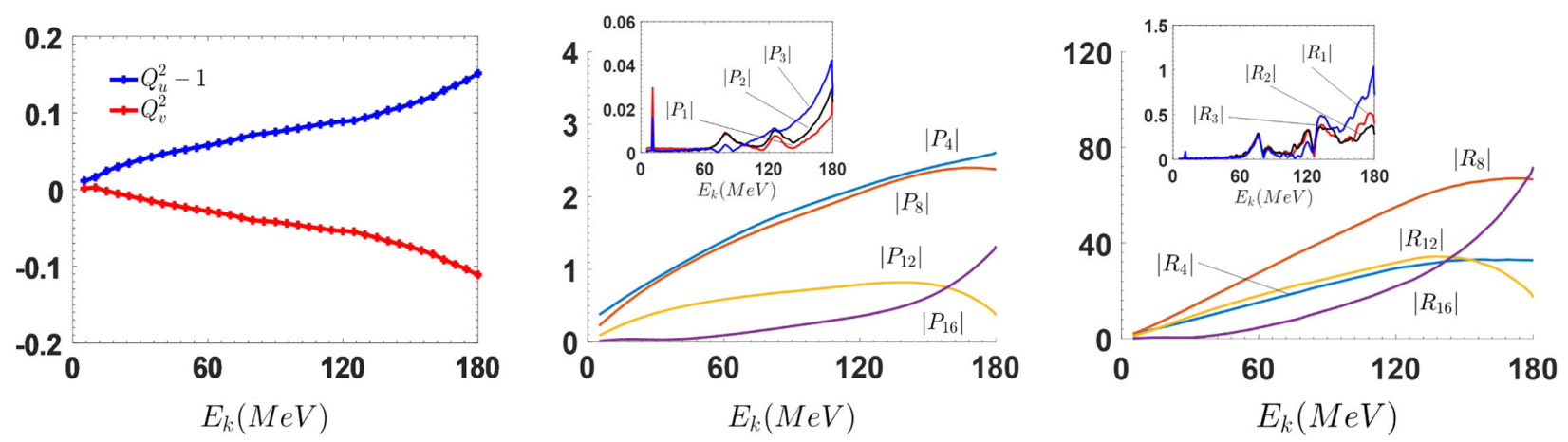

FIG. 11. Values of (left) $Q_{u}^{2}-1$ and $Q_{v}^{2}$, (middle) the Fourier coefficients of $g(\theta)$, and (right) the Fourier coefficients of $J(\theta)$ for different energy particles.

$$
\begin{gathered}
u^{\prime \prime}+Q_{u}^{2} \cdot u=-g(\theta) \cdot u, \\
v^{\prime \prime}+Q_{v}^{2} \cdot v=-h(\theta) \cdot v+J(\theta) \cdot u v .
\end{gathered}
$$

With the above procedure, the original transverse oscillation equations are transformed into Mathieu-type equations [Eqs. (A18) and (A19)]. The details of the coefficients of Eqs. (A18) and (A19) are given in Figs. 10 and 11.

[1] R. S. Zounes and R. H. Rand, Transition curves for the quasi-periodic Mathieu equation, SIAM J. Appl. Math. 58, 1094 (1998).

[2] T. Insperger and G. Stepan, Stability of the damped Mathieu equation with time delay, J. Dyn. Syst., Meas., Control 125, 166 (2003).

[3] D. Younesian, E. Esmailzadeh, and R. Sedaghati, Asymptotic solutions and stability analysis for generalized nonhomogeneous Mathieu equation, Commun. Nonlinear Sci. Numer. Simul. 12, 58 (2007).

[4] V. Ramakrishnan and B. F. Feeny, Resonances of a forced Mathieu equation with reference to wind turbine blades, J. Vib. Acoust. 134, 064501 (2012).

[5] M. H. Holmes, Introduction to Perturbation Methods (Springer Science \& Business Media, New York, 2012).

[6] K. Zhou, Y.T. Song, K. Z. Ding, J. Ge, and K. Yao, Analytical solution of transverse oscillation in cyclotron using LP method, Chin. Phys. C 42, 037001 (2018).

[7] E. Salahshoor, S. Ebrahimi, and M. Maasoomi, Nonlinear vibration analysis of mechanical systems with multiple joint clearances using the method of multiple scales, Mech. Mach. Theory 105, 495 (2016).

[8] A. H. Nayfeh and D. T. Mook, Nonlinear Oscillations (John Wiley \& Sons, New York, 2008).

[9] J.C. Li and X.C. Zhou, Asymptotic Methods in Mathematical Physics, 1st ed. (Science Press, Beijing, 1998).

[10] S.H. Chen, The Definite Quantitative Methods for Strongly Non-linear Vibration, 1st ed. (Guangdong Science and Technology Press, Guangzhou, 2004).

[11] S. Fan, Y. Li, Z. Guo, J. Li, and H.-H. Zhuang, Dynamic characteristics of resonant gyroscopes study based on the
Mathieu equation approximate solution, Chin. Phys. B 21, 050401 (2012).

[12] T. J. Zhang, H. J. Yao, J. J. Yang, J. Q. Zhong, and S. Z. An, The beam dynamics study for the CYCIAE-100 cyclotron, Nucl. Instrum. Methods Phys. Res., Sect. A 676, 90 (2012).

[13] T. J. Zhang, M. Li, J. Q. Zhong, S. Z. An, and S. M. Wei, Beam dynamics study for a small, high current $14 \mathrm{MeV}$ PET cyclotron, Nucl. Instrum. Methods Phys. Res., Sect. A 269, 2955 (2011).

[14] M. Biagini, M. Boscolo, and M. Ferrario, Beam dynamics studies for the SPARC project, in Proceedings of the 2003 Particle Accelerator Conference, Portland, OR (IEEE, New York, 2003).

[15] D. D. Caussyn, M. Ball, B. Brabson et al., Experimental studies of nonlinear beam dynamics, Phys. Rev. A 46, 7942 (1992).

[16] L. H. Yu, Analysis of nonlinear dynamics by square matrix method, Phys. Rev. Accel Beams 20, 034001 (2017).

[17] G. A. Karamysheva and S. A. Kostromin, Beam dynamics study in the $\mathrm{C} 235$ cyclotron for proton therapy, Phys. Part. Nucl. Lett. 6, 84 (2009).

[18] A. Wolski and A. T. Herrod, Explicit symplectic integrator for particle tracking in s-dependent static electric and magnetic fields with curved reference trajectory, Phys. Rev. Accel. Beams 21, 084001 (2018).

[19] M. M. Gordon, Fixed-point orbits in the vicinity of the $\mathrm{vr}=N / 3, N / 4$, and $N / 2$ resonances, Nucl. Instrum. Methods 18, 281 (1962).

[20] D. Jeon and M. M. Gordon, Coupling effects at the $\mathrm{vZ}=$ $3 / 4$ resonance in a three-sector superconducting cyclotron, Nucl. Instrum. Methods Phys. Res., Sect. A 349, 1 (1994).

[21] C. J. A. Corsten and H. L. Hagedoorn, Simultaneous treatment of betatron and synchrotron motions in circular accelerators, Nucl. Instrum. Methods Phys. Res. 212, 37 (1983).

[22] Y. Jongen, W. Beeckman, W. Kleeven, D. Vandeplassche, S. Zaremba, E. Samsonov, and N. Morozov, Numerical study of the resonance in superconducting cyclotron C400, in Proceedings of the 18th International Conference on Cyclotrons and their Applications (L.A.C. Piazza, INFN LNS, Catania, 2007).

[23] W. Kleeven and H. Hagedoorn, The influence of magnetic field imperfections on the beam quality in a $H^{-}$cyclotron, 
in Proceedings of the 13th International Conference on Cyclotrons and their Applications (World Scientific, Singapore, 1993).

[24] E. D. Courant and H. S. Snyder, Theory of the alternatinggradient synchrotron, Ann. Phys. (N.Y.) 281, 360 (2000).

[25] J. Y. Tang and B. W. Wei, Theory and Design of Cyclotrons (University of Science and Technology of China Press, Hefei, 2008).

[26] G. Karamysheva, Y. Bi, G. Chen et al., Compact Superconducting Cyclotron SC200 for proton therapy, in
Proceedings of the 21st International Conference on Cyclotrons and their Applications, Zurich, Switzerland (JACoW, Geneva, Switzerland, 2016), https://doi.org/10.18429/JACoW-Cyclotrons2016-MOP14.

[27] M. M. Gordon and D. O. Jeon, Improved formulas for calculating cyclotron orbit properties, Nucl. Instrum. Methods Phys. Res., Sect. A 301, 182 (1991).

[28] M. M. Gordon, Computation of closed orbits and basic focusing properties for sector-focused cyclotrons and the design of "cyclops", Part. Accel. 16, 39 (1984). 